\title{
Unifying Quantum and Classical Speed Limits on Observables
}

\author{
Luis Pedro García-Pintos $\odot,{ }^{1, *}$ Schuyler B. Nicholson, ${ }^{2}$ Jason R. Green ${ }^{3,4,5}$ \\ Adolfo del Campo®,$^{6,7,5}$ and Alexey V. Gorshkov $\oplus^{1}$ \\ ${ }^{1}$ Joint Center for Quantum Information and Computer Science and Joint Quantum Institute, \\ NIST/University of Maryland, College Park, Maryland 20742, USA \\ ${ }^{2}$ Department of Chemistry, Northwestern University, 2145 Sheridan Road, Evanston, Illinois 60208, USA \\ ${ }^{3}$ Department of Chemistry, University of Massachusetts Boston, Boston, Massachusetts 02125, USA \\ ${ }^{4}$ Center for Quantum and Nonequilibrium Systems, University of Massachusetts Boston, \\ Boston, Massachusetts 02125, USA \\ ${ }^{5}$ Department of Physics, University of Massachusetts Boston, Boston, Massachusetts 02125, USA \\ ${ }^{6}$ Department of Physics and Materials Science, University of Luxembourg, \\ L-1511 Luxembourg, G. D. Luxembourg \\ ${ }^{7}$ Donostia International Physics Center, E-20018 San Sebastián, Spain
}

(Received 1 September 2021; revised 18 December 2021; accepted 22 December 2021; published 28 February 2022)

The presence of noise or the interaction with an environment can radically change the dynamics of observables of an otherwise isolated quantum system. We derive a bound on the speed with which observables of open quantum systems evolve. This speed limit is divided into Mandelstam and Tamm's original time-energy uncertainty relation and a time-information uncertainty relation recently derived for classical systems, and both are generalized to open quantum systems. By isolating the coherent and incoherent contributions to the system dynamics, we derive both lower and upper bounds on the speed of evolution. We prove that the latter provide tighter limits on the speed of observables than previously known quantum speed limits and that a preferred basis of speed operators serves to completely characterize the observables that saturate the speed limits. We use this construction to bound the effect of incoherent dynamics on the evolution of an observable and to find the Hamiltonian that gives the maximum coherent speedup to the evolution of an observable.

DOI: 10.1103/PhysRevX.12.011038

\section{INTRODUCTION}

How quickly can the expectation value $\langle A\rangle$ of an observable change as a quantum system evolves in the presence of an environment? What properties of the system allow for fast evolution of a physical quantity? How sensitive is the speed of an observable's evolution to the effects of an environment? We probe these questions by deriving speed limits on observables, i.e., uncertainty relations that bound the rate of change of $\langle A\rangle$. Our main results rely on discriminating the "quantumlike" coherent contributions and the "classical-like" incoherent contributions to the evolution of an open quantum system. This allows us to derive lower bounds on speed, as well as upper

\footnotetext{
* Corresponding author. lpgp@umd.edu

Published by the American Physical Society under the terms of the Creative Commons Attribution 4.0 International license. Further distribution of this work must maintain attribution to the author(s) and the published article's title, journal citation, and DOI.
}

Subject Areas: Quantum Physics, Quantum Information Statistical Physics

bounds on speed, which are tighter than those previously considered. In this way, we also unify-and improve upon-previously known quantum and classical speed limits on observables.

Mandelstam and Tamm first derived a bound on the speed for quantum systems evolving unitarily under a Hamiltonian $H$ [1]. They proved that

$$
\left|\frac{d\langle A\rangle}{d t}\right| \leq 2 \Delta A \Delta H
$$

where $\Delta A:=\sqrt{\left\langle A^{2}\right\rangle-\langle A\rangle^{2}}$ and $\Delta H$ are the standard deviations of the observable and the Hamiltonian, respectively (units are such that $\hbar=1$ ). This result bounds the speed of evolution of any physical quantity of an isolated quantum system. Mandelstam and Tamm further considered the projection onto the initial state of the system, $A:=\left|\psi_{0}\right\rangle\left\langle\psi_{0}\right|$, as an observable of interest. Equation (1) then implies a bound on how fast the state of the system evolves. They proved that the minimum time $\tau^{\perp}$ for a system to evolve between two orthogonal states satisfies $\tau^{\perp} \geq \pi /(2 \Delta H)$. This gives an ultimate limit to the speed of 
evolution in the system: A minimum time has to elapse for the state of the system to evolve into a distinguishable state, $t \geq \tau^{\perp}$.

Since then, the focus on state distinguishability instead of observables has been predominant, with most works adopting a metric in Hilbert space and deriving bounds on its rate of change. For instance, some studies have focused on alternative bounds to that of Mandelstam and Tamm [2-5], involving various metrics [6-17] and covering more general dynamical regimes [18-21]. In this way, quantum speed limits have found applications in a range of topics, including quantum control [22-25], limits to computation [2,26,27], parameter estimation [28-30], quantum thermodynamics [31,32], quantum annealing [33], quantum information theory [34-37], and the dynamics of many-body [38,39] and open quantum systems [40]. Quantum speed limits have even been extended to classical settings by using metrics in the space of probability distributions [41-44]. Some studies have deviated from the focus on speed limits in Hilbert space, deriving bounds on quantum thermodynamic processes [45-47]. However, metric-based speed limits remain prevalent [48].

Approaches based on metrics in Hilbert space have a shortcoming: While any pair of orthogonal states are distinguishable under some measurement, oftentimes relevant observables remain unchanged. For example, consider an ensemble of two-level systems that evolve from state $|\uparrow \uparrow \uparrow \ldots \uparrow \uparrow\rangle$ to state $|\uparrow \uparrow \uparrow \ldots \uparrow \downarrow\rangle$ over a time $\tau^{\perp}$. All distances between these states achieve their maximum value, but interesting observables such as the magnetizations $\sum_{j} \sigma_{j}^{z}$ or $\sum_{j} \sigma_{j}^{x}$ barely change (here, $\sigma^{\alpha}$ are Pauli matrices). Observables can be thought of as "filter functions," sensitive only to restricted parts of the dynamics of the state. While bounds on $\tau^{\perp}$ provide information about the fastest evolving Hermitian operators, they may not reflect the dynamics of experimentally relevant physical observables [49]. This situation is exacerbated by the fact that the speed limits of different metrics can vary significantly $[10,13]$. We see this as a prime example that highlights the need for speed limits on physically grounded quantities physically grounded quantities of direct relevance of direct relevance to experimental measurements.

In this work, we address the shortcomings of derivations of speed limits for distances in Hilbert space. We do so by deriving limits on the evolution of arbitrary observables for arbitrary differentiable dynamics. This extends the original derivation by Mandelstam and Tamm, which was restricted to isolated quantum systems. In doing so, we also generalize Mandelstam and Tamm's version of the quantum timeenergy uncertainty relation to open quantum systems. This result is the quantum analogue of a time-information uncertainty relation recently introduced for stochastic dynamics of classical systems [50], in which the maximal rate of change of an observable depends on (classical) fluctuations in the observable and fluctuations in the surprisal rate of the system.

The remainder of the paper is organized as follows. Section II shows a general bound on the rate of change of observables for open quantum systems in terms of the quantum Fisher information and discusses connections to the quantum Cramér-Rao bound. The core results of this paper are contained in Sec. III, where we derive strictly tighter upper bounds than those in Sec. II by decomposing the dynamics of a quantum system in terms of coherent and incoherent contributions. This technique also allows us to derive, for the first time, lower bounds on the speed of observables. We show in Sec. IV how to connect these bounds to energy uncertainties of the system and derive bounds on energy and entropy rates, providing a simple proof of the "small incremental entangling theorem." Section V focuses on the tightness of the speed limits obtained in Secs. II and III. We derive limits to the total change of an observable in Sec. VI, where we use them to bound the integrated effects from incoherent dynamics on an open quantum system. In Sec. VII, we provide conclusions and a discussion. Finally, in the Appendixes, we present derivations of these results.

\section{SPEED LIMITS ON OBSERVABLES IN OPEN QUANTUM SYSTEMS}

Let $\rho_{t}$ denote an arbitrary density matrix of a quantum system, possibly characterizing a statistical mixture over pure states. Its dynamics can be formally expressed by the symmetric logarithmic derivative $L$, implicitly defined by $\left(d \rho_{t} / d t\right)=: \frac{1}{2}\left\{L, \rho_{t}\right\} \quad[28,51-53]$, where $\{A, B\}=A B+B A$ denotes an anticommutator. Using this equation of motion, we show (Appendix A) that the expectation value $\langle A\rangle=\operatorname{Tr}\left(A \rho_{t}\right)$ evolves according to a generalized form of the Ehrenfest equation,

$$
\frac{d\langle A\rangle}{d t}=\operatorname{cov}(L, A)+\left\langle\frac{d A}{d t}\right\rangle=: \dot{a}+\left\langle\frac{d A}{d t}\right\rangle,
$$

where $\operatorname{cov}(A, L):=\frac{1}{2} \operatorname{Tr}\left(\rho_{t}\{A, L\}\right)-\langle A\rangle\langle L\rangle$ is the symmetrized covariance. Here, we distinguish between the term $\langle d A / d t\rangle$, which stems from any explicit time dependence of the observable, and $\dot{a}:=\operatorname{Tr}\left[A\left(d \rho_{t} / d t\right)\right]=\operatorname{cov}(L, A)$, which depends on the rate of change of the state. Note that for time-dependent observables, $a$ is not a state function but is instead defined through the path-dependent integral $a:=\int \dot{a} d t$. An analogy is that of heat and work in thermodynamics, whose total changes are defined via the infinitesimal changes of a given process and cannot generally be defined only in terms of the initial and final states. In fact, if $A=H(t)$ is the Hamiltonian of the system, $\dot{a}$ and $\langle\dot{A}\rangle$ correspond to heat and work exchanges in 
quantum thermodynamics $[54,55]$. For observables without explicit time dependence, one simply has $\dot{a}=d\langle A\rangle / d t$.

An equation of motion analogous to (2) has been proven for classical stochastic systems [50] and is known as the Price equation in evolutionary biology [56,57]. Applying the Cauchy-Schwarz inequality, we can derive the following upper bound on the change in the expectation value due to state changes, $\dot{a}:=\operatorname{Tr}\left[A\left(d \rho_{t} / d t\right)\right]=\operatorname{cov}(L, A)$, which generalizes the Mandelstam and Tamm speed limit on observables:

$$
|\dot{a}|=|\operatorname{cov}(L, A)| \leq \Delta A \Delta L=\Delta A \sqrt{\mathcal{I}_{F}} .
$$

Here, $\mathcal{I}_{F}:=(\Delta L)^{2}$ is the quantum Fisher information. For a density matrix with a spectral decomposition $\rho_{t}=\sum_{j} p_{j}|j\rangle\langle j|$, it is given by $[28,53,58]$

$$
\mathcal{I}_{F}=(\Delta L)^{2}=2 \sum_{j k}^{d} \frac{\left|\left\langle j\left|\frac{d}{d t} \rho_{t}\right| k\right\rangle\right|^{2}}{p_{j}+p_{k}} .
$$

The definition of $\mathcal{I}_{F}$ and the proof of Eq. (3) assume differentiable dynamics and that $p_{j} \neq 0 \forall j$ [59]. In order to ease notation, we omit explicit time dependence of $\mathcal{I}_{F}, p_{j}$, $\{|j\rangle\}, L$, and $A$.

Equation (3) shows that the uncertainty of an observable and the quantum Fisher information limit the speed with which the mean of the observable evolves. The Fisher information $\mathcal{I}_{F}$ originates from parameter estimation theory, where it bounds the uncertainty with which a parameter $-t$ in this case — can be determined [51-53,58,60]. Speed limits in Hilbert space $[4,5,10,18,61]$ and speed limits for observables in closed systems $[28,46]$ have also been linked to bounds from parameter estimation theory. In fact, when $A$ has no explicit time dependence, Eq. (3) can be derived from the quantum Cramér-Rao bound by restricting to functions of $\langle A\rangle$ as (typically suboptimal) estimators of the parameter $t$ (Appendix K). Note, though, that the quantum Cramér-Rao bound encompasses arbitrary estimators constructed from all possible measurements that can be performed on the system. While an optimal choice of such an estimator saturates the quantum Cramér-Rao bound, there can exist tighter bounds for other given estimators. We will exploit this in the next section to derive speed limits on $\langle A\rangle$ that are tighter than the speed limit (3) implied by the quantum Cramér-Rao bound [62].

For a given speed $\dot{a}$, the uncertainty bound (3) implies a direct trade-off between how certain an observable is and the Fisher information $\mathcal{I}_{F}$. Roughly speaking, $\mathcal{I}_{F}$ is a measure of stationarity in the system [63,64]: A small value implies a weak change of $\rho_{t}$ in time, which hinders the rate of change of any observable. Similarly, $\Delta A=0$ implies a state $\rho_{t}$ supported on the subspace spanned by eigenvectors $\left\{\left|\lambda_{a}^{l}\right\rangle\right\}$ corresponding to a single eigenvalue $\lambda_{a}$ of $A$. Then, the function $\operatorname{Tr}\left(\rho_{t} \sum_{l}\left|\lambda_{a}^{l}\right\rangle\left\langle\lambda_{a}^{l}\right|\right)=\langle A\rangle / \lambda_{a}=1$ is at a maximum, implying a null rate of change, which translates into $\dot{a}=0$. On the other extreme, fast observable dynamics requires large fluctuations and large Fisher information.

Following Mandelstam and Tamm, we identify the characteristic timescale $\tau_{a}:=\Delta A /|\dot{a}|$ over which the expectation value of an observable changes by a standard deviation [1]. Combining this definition with the inequality (1), Mandelstam and Tamm established the time-energy uncertainty relation $\tau_{A} \Delta H \geq 1 / 2$, valid for isolated systems evolving with a constant Hamiltonian $H[1,65]$; see Refs. [28,66,67] as well. For pure states, $\mathcal{I}_{F}=4(\Delta H)^{2}$, and Mandelstam-Tamm's time-energy uncertainty can be reexpressed as $\tau_{A} \sqrt{\mathcal{I}_{F}} \geq 1$. Bound (3) extends this to states following arbitrary differentiable dynamics,

$$
\tau_{A} \sqrt{\mathcal{I}_{F}} \geq 1,
$$

and constitutes a time-information uncertainty relation that holds universally for quantum systems.

It is worth noting that uncertainty relations are not purely a feature of quantum mechanics. In fact, Ref. [50] shows that a bound analogous to Eq. (5) holds for classical stochastic systems. How, then, does the interplay of quantum and classical dynamics contribute to the speed at which an observable can evolve?

\section{SPEED LIMITS FOR COHERENT AND INCOHERENT DYNAMICS}

The state $\rho_{t}$ of a quantum system evolving under arbitrary differentiable trace-preserving dynamics can be written as $\rho_{t}=U_{t} \chi_{t} U_{t}^{\dagger}$. The unitary operator $U_{t}$ connects the time-dependent eigenbasis of $\rho_{t}$ to the eigenbasis $\left\{|j\rangle_{0}\right\}$ of the initial state by $|j\rangle=U_{t}|j\rangle_{0}$, and $\chi_{t}:=\sum_{j} p_{j}|j\rangle_{00}\langle j|$ is a diagonal density matrix with the same eigenvalues as $\rho_{t}$. Defining the Hermitian operator $H_{t}:=i\left(d U_{t} / d t\right) U_{t}^{\dagger}$ as the "Hamiltonian," one can express the evolution of $\rho_{t}$ as [68-70]

$$
\frac{d}{d t} \rho_{t}=-i\left[H_{t}, \rho_{t}\right]+U_{t} \frac{d \chi_{t}}{d t} U_{t}^{\dagger} .
$$

This equation describes the dynamics of any quantum system with a continuous physical evolution where probability is conserved, including non-Markovian dynamics [71] and nonlinear dynamics stemming from continuous monitoring [72] or balanced gain and loss [70]. In the widely relevant case when the system obeys a Lindblad equation for Lindblad operators $\Gamma_{\alpha}$ with rates $\gamma_{\alpha}$ that cause incoherent dynamics, one has $U_{t}\left(d \chi_{t} / d t\right) U_{t}^{\dagger}=$ $\sum_{\alpha} \gamma_{\alpha}\left(\Gamma_{\alpha} \rho \Gamma_{\alpha}^{\dagger}-\frac{1}{2}\left\{\Gamma_{\alpha}^{\dagger} \Gamma_{\alpha}, \rho\right\}\right)$. (Note that Lindblad operators can also induce unitary dynamics, in which case they would contribute to $H_{t}[73,74]$.)

The first term in Eq. (6) represents coherent, entropypreserving evolution. The second is the incoherent term, 
which corresponds to changes in the state's eigenvalues and therefore in the entropy of the system. Note that the case with no coherent contribution, $H_{t}=0$, gives rise to a description equivalent to a probability distribution $\left\{p_{j}\right\}$ following classical stochastic dynamics [75]. In contrast, even if coherence is not a uniquely quantum trait [76], coherent dynamics due to $H_{t} \neq 0$ is characteristic of quantum systems. In this sense, one could broadly identify the coherent and incoherent terms to correspond to "quantum" and "classical" contributions to the evolution, respectively $[10,69]$. This identification is further supported by the fact that, if the eigenstates $|j\rangle$ involved in purely incoherent dynamics are classical (e.g., bit strings representing product states of computational basis states of twolevel systems), the resulting incoherent dynamics is indeed classical. At the same time, one should take the classical label for incoherent dynamics with a grain of salt since eigenstates $|j\rangle$ can be highly nontrivial, in which case incoherent dynamics can also be quantum-for instance, driving product states into entangled ones [77].

To extend the coherent-incoherent separation to the observable of interest $A:=A_{C}+A_{I}$, we define the relevant components to the evolution of the observable for a system with purely coherent $\left(\dot{\chi}_{t}=0\right)$ or purely incoherent $\left(H_{t}=0\right)$ dynamics,

$$
A_{C}:=\sum_{j \neq k}^{d} A_{j k}|j\rangle\langle k| \quad \text { and } \quad A_{I}:=\sum_{j}^{d} A_{j j}|j\rangle\langle j| .
$$

In this separation, we take the time-dependent basis $\{|j\rangle\}$ that diagonalizes state $\rho_{t}$. The coherent-incoherent division is thus relative to the state of the system.

As a final ingredient to our construction, we define Hermitian operators $L_{C}$ and $L_{I}$, with $L=L_{C}+L_{I}$, which will characterize the speed due to coherent and incoherent dynamics:

$$
\begin{aligned}
& L_{C}:=-2 i \sum_{j \neq k}^{d} \frac{\left\langle j\left|\left[H_{t}, \rho_{t}\right]\right| k\right\rangle}{\left(p_{j}+p_{k}\right)}|j\rangle\langle k|, \\
& L_{I}:=\sum_{j}^{d} \frac{d \ln p_{j}}{d t}|j\rangle\langle j| .
\end{aligned}
$$

With these operators, one can separate the quantum Fisher information into coherent and incoherent contributions $\mathcal{I}_{F}=\mathcal{I}_{F}^{C}+\mathcal{I}_{F}^{I}$ (Appendix C):

$$
\begin{aligned}
& \mathcal{I}_{F}^{C}:=\left(\Delta L_{C}\right)^{2}=2 \sum_{j \neq k}^{d} \frac{\left|\left\langle j\left|\left[H_{t}, \rho_{t}\right]\right| k\right\rangle\right|^{2}}{p_{j}+p_{k}}, \\
& \mathcal{I}_{F}^{I}:=\left(\Delta L_{I}\right)^{2}=\sum_{j}^{d} p_{j}\left(\frac{d}{d t} \ln p_{j}\right)^{2} .
\end{aligned}
$$

Note that $\mathcal{I}_{F}^{I}$ is the classical Fisher information of the probability distribution $\left\{p_{j}\right\}[78,79]$, which also admits an interpretation in terms of the variance in the surprisal rate $\left\{\left(L_{I}\right)_{j}=-(d / d t) \ln p_{j}\right\}$ associated with the eigenvalue distribution $\left\{p_{j}\right\}$ [80]. Meanwhile, $\mathcal{I}_{F}^{C}$ is the quantum Fisher information for a system evolving unitarily. For pure states, $\mathcal{I}_{F}^{C}=4\left(\Delta H_{t}\right)^{2}$ [53].

This construction, which separates the change of an observable $A$ into coherent and incoherent contributions, allows for the derivation of bounds that are tighter than the speed limit (3). We prove in Appendix $\mathrm{C}$ that, for differentiable dynamics, $\dot{a}_{C}:=\operatorname{Tr}\left[A_{C}\left(d \rho_{t} / d t\right)\right]=\operatorname{cov}\left(A_{C}, L_{C}\right)$ and $\dot{a}_{I}:=\operatorname{Tr}\left[A_{I}\left(d \rho_{t} / d t\right)\right]=\operatorname{cov}\left(A_{I}, L_{I}\right)$. Therefore,

$$
\begin{gathered}
\left|\dot{a}_{C}\right|=\left|\operatorname{cov}\left(A_{C}, L_{C}\right)\right| \leq \Delta A_{C} \sqrt{\mathcal{I}_{F}^{C}}, \\
\left|\dot{a}_{I}\right|=\left|\operatorname{cov}\left(A_{I}, L_{I}\right)\right| \leq \Delta A_{I} \sqrt{\mathcal{I}_{F}^{I}}
\end{gathered}
$$

set bounds on the rate of change of an observable that isolate the role played by coherent and incoherent dynamics. Equation (10b) is identical to the one derived in Ref. [50] for classical stochastic systems, where $-\left(L_{I}\right)_{j}$ corresponds to the surprisal rates. This coherent-incoherent decomposition and the corresponding bounds constitute one of the main results of the paper.

These results provide a foundation for a number of speed limits on observables. The reverse triangle inequality [81] yields a lower bound on the speed $\dot{a}=\operatorname{Tr}\left[A\left(d \rho_{t} / d t\right)\right]=\dot{a}_{C}+\dot{a}_{I}$, i.e., a coherent-incoherent lower speed limit

$$
|\dot{a}| \geq \max \left\{\left|\dot{a}_{C}\right|-\Delta A_{I} \sqrt{\mathcal{I}_{F}^{I}},\left|\dot{a}_{I}\right|-\Delta A_{C} \sqrt{\mathcal{I}_{F}^{C}}\right\},
$$

made possible by the division of the system dynamics into two contributions. To the best of our knowledge, this is the first derivation of general lower speed limits on the evolution of open quantum systems.

Equation (10) also implies a new upper bound singling out additive contributions from the uncertainties of the coherent and incoherent parts $A_{C}$ and $A_{I}$ of the observable,

$$
\begin{aligned}
|\dot{a}| & \leq \min \left\{\left|\dot{a}_{C}\right|+\Delta A_{I} \sqrt{\mathcal{I}_{F}^{I}},\left|\dot{a}_{I}\right|+\Delta A_{C} \sqrt{\mathcal{I}_{F}^{C}}\right\} \\
& \leq \Delta A_{C} \sqrt{\mathcal{I}_{F}^{C}}+\Delta A_{I} \sqrt{\mathcal{I}_{F}^{I}} .
\end{aligned}
$$

This bound limits the speed of systems following coherent quantum dynamics, as well as systems following incoherent classical dynamics: a coherent-incoherent speed limit. While the speed limit (3) is saturated by a properly chosen observable (i.e., an optimal estimator in the context of the quantum Cramér-Rao bound from parameter estimation theory), we show in Sec. V below that the 
coherent-incoherent speed limit (12) is tighter for any fixed $A$, imposing stricter constraints on the speed of evolution.

Two limiting cases demonstrate the scope of the upper bound. For purely coherent evolution, constant probabilities $\dot{p}_{j}(t)=0$ imply $\mathcal{I}_{F}^{C}=0$, in which case the coherentincoherent speed limit recovers the Mandelstam-Tamm bound for observables, generalized to allow for an explicit time dependence in the Hamiltonian. In the opposite extreme of purely incoherent dynamics, $H_{t}=0$, it recovers the classical speed limit recently derived in Ref. [50] for classical stochastic dynamics.

More generally, consider a system in state $\rho_{t}$ with dynamics that results in $\mathcal{I}_{F}^{I}$ and $\mathcal{I}_{F}^{C}$. The coherent-incoherent speed limits tell us that some observables evolve following incoherent dynamics characterized by $\mathcal{I}_{F}^{I}$, while others follow the coherent dynamics with $\mathcal{I}_{F}^{C}$. The evolution of observables with $\Delta A_{C}=\sqrt{\sum_{j \neq k} p_{j}\left|A_{j k}\right|^{2}}=0$, which commute with the state, is guided by the incoherent contribution to the evolution. Meanwhile, observables with $\Delta A_{I}=0$ are driven solely by the Hamiltonian.

The coherent-incoherent partition of an observable motivates the definition of characteristic timescales $\tau_{A_{C}}:=$ $\Delta A_{C} /\left|\dot{a}_{C}\right|$ and $\tau_{A_{I}}:=\Delta A_{I} /\left|\dot{a}_{I}\right|$ for $A_{C}$ and $A_{I}$, respectively, over which each of them change by their standard deviations. From Eq. (10), their time-information uncertainty bounds are

$$
\tau_{A_{C}} \sqrt{\mathcal{I}_{F}^{C}} \geq 1 \quad \text { and } \quad \tau_{A_{I}} \sqrt{\mathcal{I}_{F}^{I}} \geq 1
$$

The first bound generalizes Mandelstam-Tamm's timeenergy uncertainty relation to the coherent component of the evolution. The second bound generalizes the classical time-information uncertainty bound from Ref. [50] to the incoherent contribution to the dynamics of an observable for a quantum system with arbitrary differentiable dynamics. Note, though, that these apply to general regimes in which an open quantum system evolves under both coherent and incoherent dynamics. They provide alternate uncertainty relations to the quantum time-information uncertainty bound (5) that single out coherent and incoherent characteristic timescales.

\section{SPEED LIMITS IN TERMS OF ENERGY VARIANCES}

One may be interested in bounds that depend on physical quantities that are experimentally more accessible than the different contributions to the Fisher information central to our results above. If we assume that the source of nonunitary dynamics is entanglement with an environment via a Hamiltonian $H_{t}^{\text {int }}$ that includes all terms with support on both the system and the environment, it holds that

$$
\mathcal{I}_{F}^{C} \leq 4\left(\Delta H_{t}\right)^{2} \quad \text { and } \quad \mathcal{I}_{F}^{I} \leq 4\left(\Delta H_{t}^{\mathrm{int}}\right)^{2} .
$$

The standard deviation $\Delta H_{t}^{\mathrm{int}}$ is calculated in the joint state of the system and the environment. The bound in Eq. (14) on $\mathcal{I}_{F}^{C}$ was proven by Braunstein and Caves in Ref. [28]. We prove the new (loose) bound in Eq. (14) on $\mathcal{I}_{F}^{I}$ in Appendix D. When combined with the coherent-incoherent speed limits (12) and (11), Eq. (14) implies upper and lower bounds on $|\dot{a}|$ in terms of energy uncertainties.

Certain physical quantities naturally evolve solely under incoherent dynamics. The von Neumann entropy $S:=-\operatorname{Tr}\left(\rho_{t} \ln \rho_{t}\right)$ of a system is one example. Using $\dot{S}=-\operatorname{Tr}\left(d \rho_{t} / d t \ln \rho_{t}\right)$ [82] and choosing $A_{I}=-\ln \rho_{t}$ in Eq. (10) gives a bound

$$
|\dot{S}| \leq \Delta S \sqrt{\mathcal{I}_{F}^{I}} \leq 2 \Delta S \Delta H_{t}^{\mathrm{int}}
$$

set by the variance $(\Delta S)^{2}:=\operatorname{Tr}\left(\rho_{t}\left(\ln \rho_{t}\right)^{2}\right)-S^{2}$ of the surprisal operator $\left[-\ln \rho_{t}\right]$, whose expectation value is the von Neumann entropy. In other words, an uncertainty relation bounds the change in entropy of any open quantum system in terms of fluctuations in energy and in surprisal $\left[-\ln \rho_{t}\right](\Delta S$ also plays the interesting role of determining the possible transitions between states of a quantum system [83]). Using $\Delta S \leq \sqrt{(\ln (d-1))^{2} / 4+1}[83,84]$ and $\Delta H_{t}^{\text {int }} \leq\left\|H_{t}^{\text {int }}\right\|$, where $\left\|H_{t}^{\text {int }}\right\|$ is the operator norm, we recover the small incremental entangling theorem $[85,86]$, $|\dot{S}| \lesssim \ln d\left\|H_{t}^{\text {int }}\right\|$, in the case of no ancillary systems (Appendix E).

Even though the Hamiltonian is generally not of the form of $A_{C}$, since it need not commute with $\rho_{t}$, a direct calculation with Eq. (6) shows that the energy of the system changes solely due to the time dependence of $H_{t}$ or under the incoherent contribution to the dynamics. Thus, from Eqs. (10) and (14), it holds that

$$
\left|\operatorname{Tr}\left(\frac{d \rho_{t}}{d t} H_{t}\right)\right| \leq \Delta H_{C} \sqrt{\mathcal{I}_{F}^{C}} \leq \Delta H_{t} \Delta H_{t}^{\mathrm{int}},
$$

where $H_{C}$ is the diagonal component of $H_{t}$ as defined in Eq. (7) and we use $\Delta H_{C} \leq \Delta H_{t}$. This result sets bounds on what is typically identified as the heat flux in the quantum thermodynamics of open systems [54,55] [87]. This new speed limit is a quantum analogue of the bound derived in Ref. [50] for classical stochastic thermodynamics.

\section{SATURATION AND TIGHTNESS OF THE SPEED LIMITS}

Here, we focus on the tightness of the main, new, coherent-incoherent speed limit (12) and how it compares to the speed limit (3). We find that the former provides a strictly tighter constraint on the rate of change of observables than the speed limit implied by the quantum CramérRao bound. To see this, we use $\mathcal{I}_{F}=\mathcal{I}_{F}^{C}+\mathcal{I}_{F}^{I}$ and $(\Delta A)^{2}=\left(\Delta A_{C}\right)^{2}+\left(\Delta A_{I}\right)^{2}$ to prove that (Appendix F) 
$\frac{\Delta A \sqrt{\mathcal{I}_{F}}}{\Delta A_{C} \sqrt{\mathcal{I}_{F}^{C}}+\Delta A_{I} \sqrt{\mathcal{I}_{F}^{I}}}=\sqrt{1+\frac{\left(\Delta A_{C} \sqrt{\mathcal{I}_{F}^{I}}-\Delta A_{I} \sqrt{\mathcal{I}_{F}^{C}}\right)^{2}}{\left(\Delta A_{C} \sqrt{\mathcal{I}_{F}^{C}}+\Delta A_{I} \sqrt{\mathcal{I}_{F}^{I}}\right)^{2}}}$,

which quantifies the improvement that the coherentincoherent speed limit provides. The two bounds coincide when $\Delta A_{C} \sqrt{\mathcal{I}_{F}^{I}}=\Delta A_{I} \sqrt{\mathcal{I}_{F}^{C}}$. This condition occurs for pure states $\rho_{t}=|k\rangle\langle k|$ with purely coherent dynamics $\left[\left(\Delta A_{I}\right)^{2}=\sum_{j} p_{j} A_{j j}^{2}-\left(\sum_{j} p_{j} A_{j j}\right)^{2}=0\right.$ and $\left.\mathcal{I}_{F}^{I}=0\right]$, in which case bounds (12) and (3) coincide with the one by Mandelstam and Tamm [1]. It also occurs for diagonal observables with purely incoherent dynamics $\left(\Delta A_{C}=0\right.$ and $\mathcal{I}_{F}^{C}=0$ ), in which case, both bounds coincide with the classical one derived in Ref. [50]. In contrast, whenever $\Delta A_{C} \sqrt{\mathcal{I}_{F}^{I}} \neq \Delta A_{I} \sqrt{\mathcal{I}_{F}^{C}}$, our new coherent-incoherent speed limit (12) is tighter.

Taking advantage of the fact that $\dot{a}=\operatorname{cov}(A, L)$, $\dot{a}_{C}=\operatorname{cov}\left(A_{C}, L_{C}\right)$, and $\dot{a}_{I}=\operatorname{cov}\left(A_{I}, L_{I}\right)$, one can identify observables that evolve at the limiting speeds. The speed limit (3) saturates when $\operatorname{cov}(A, L)=\Delta A \Delta L$, which is the case for observables $A \propto L$. Similarly, observables such that $A_{C} \propto L_{C}$ and $A_{I} \propto L_{I}$ saturate the coherent and incoherent speed limits (10) [89]. This property defines the role of Hermitian operators $L_{C}$ and $L_{I}$, evaluated at $\rho_{t}$, as observables that evolve at their speed limits. We can also see how saturation of the coherent-incoherent speed limit can occur in cases when the speed limit (3) is looser: An operator $A=\alpha_{C} L_{C}+\alpha_{I} L_{I}$ saturates the former but not the latter except when $\alpha_{C}=\alpha_{I}$.

In fact, the "fast" coherent and incoherent operators $L_{C}$ and $L_{Q}$ are orthogonal to each other with respect to the inner product defined by the symmetrized covariance, $\operatorname{cov}\left(L_{C}, L_{I}\right)=0$. Thus, they form part of an orthogonal basis of speed operators $\left\{L_{C}, L_{I}, L_{0}^{1}, \ldots, L_{0}^{d^{2}-2}\right\}$ that spans the space of Hermitian operators. Since evolution occurs only under coherent or incoherent dynamics, and $\operatorname{cov}\left(L_{C}, L_{0}^{n}\right)=\operatorname{cov}\left(L_{I}, L_{0}^{n}\right)=0$ by construction, the "still" operators $\left\{L_{0}^{n}\right\}$ do not evolve. Then, any observable expressed in terms of the preferred basis as $A=\alpha_{C} L_{C}+$ $\alpha_{I} L_{I}+\sum_{n} \alpha_{n} L_{0}^{n}$ evolves with a speed $\dot{a}=\alpha_{C} \mathcal{I}_{F}^{C}+\alpha_{I} \mathcal{I}_{F}^{I}$.

We can use this construction to understand the situations in which the reverse triangle inequality used to derive the lower speed limit (11) saturates. The bound $|\dot{a}| \geq\left|\dot{a}_{C}\right|-$ $\Delta A_{I} \sqrt{\mathcal{I}_{F}^{I}}$ is saturated if $A_{I}=-\alpha_{I} L_{I}$ with $\alpha_{I} \geq 0$, while $|\dot{a}| \geq\left|\dot{a}_{I}\right|-\Delta A_{C} \sqrt{\mathcal{I}_{F}^{C}}$ saturates for $A_{C}=-\alpha_{C} L_{C}$ with $\alpha_{C} \geq 0$. Once again, the preferred operator basis that contains $L_{C}$ and $L_{I}$ serves to characterize the speed of an observable and how far it is from saturating the upper and lower speed limits.

The following example illustrates how to construct observables that saturate the coherent and incoherent bounds. Consider a qubit with a Hamiltonian $H=$ $(\omega / 2) \sigma_{y}$ and with incoherent dynamics driven by dephasing along $\sigma_{z}$ with a rate $\kappa$, described by $U_{t}\left(d \chi_{t} / d t\right) U_{t}^{\dagger}=$ $-\kappa\left[\sigma_{z},\left[\sigma_{z}, \rho_{t}\right]\right]$ in Eq. (6). Let us consider the case when the qubit starts with $y=\operatorname{Tr}\left(\rho_{t} \sigma_{y}\right)=0$ (Fig. 1, left column). The state of the qubit is parametrized as $\rho_{t}=$ $\left(\mathbb{1}+x \sigma_{x}+z \sigma_{z}\right) / 2$, where $x=\operatorname{Tr}\left(\rho_{t} \sigma_{x}\right)$ and $z=\operatorname{Tr}\left(\rho_{t} \sigma_{z}\right)$ are real numbers such that $x^{2}+z^{2} \leq 1$. Since the generators of dynamics preserve $y=0$, we have that $\operatorname{cov}\left(L_{C}, \sigma_{y}\right)=$ $\operatorname{cov}\left(L_{I}, \sigma_{y}\right)=0$ (Appendix G). Then, $\left\{L_{C}, L_{I}, \sigma_{y}, \mathbb{1}\right\}$ forms a complete basis of Hermitian operators, and since observables $\sigma_{x}$ and $\sigma_{y}$ are orthogonal to $\mathbb{1}$ and $\sigma_{y}$, they can be written as $\sigma_{\{z, x\}}=\alpha_{C}^{\{z, x\}} L_{C}+\alpha_{I}^{\{z, x\}} L_{I}$. This means that the coherent and incoherent terms of observables $\sigma_{x}$ and $\sigma_{z}$ evolve at the speed limits allowed by their respective bounds (10). For the case of unitary dynamics $(\kappa=0)$, this also means that the speed limit (3) saturates. However, when $\kappa \neq 0$, the latter is loose except when $\alpha_{Q}^{\{z, x\}}=\alpha_{C}^{\{z, x\}}$. Finally, saturation of the coherent-incoherent speed limits (12) and (11) depends on the relative signs of $\alpha_{C}^{\{z, x\}}$ and $\alpha_{I}^{\{z, x\}}$. Instead, if the system starts with $y \neq 0$, the fast operators have components on $\left\{\sigma_{x}, \sigma_{y}, \sigma_{z}\right\}$, which means that observables $\sigma_{\{x, y, z\}}$ do not have expressions solely in terms of the fast operators $L_{C}$ and $L_{I}$. Thus, the coherent-incoherent speed limits are typically not saturated in this case (Fig. 1, right column).

These observable-dependent bounds can vary significantly from one observable to another for a given system, as shown by the bounds on the speeds of $\sigma_{x}$ and $\sigma_{z}$ that Fig. 1 depicts. This example illustrates the spirit of our initial aim: to find bounds that better capture the speed of physical observables than speed limits in Hilbert space. The bounds obtained from the coherent-incoherent decomposition of the dynamics capture the dynamics better than those derivable from the quantum Cramér-Rao bound.

\section{BOUNDS ON INTEGRATED QUANTITIES}

The Fisher information has interesting connections to the geometry of the space of probability distributions and of state space $[18,53,61,67,90-93]$. For small changes in $t$, the quantum Fisher information is related to the Bures distance $D_{B}\left(\rho_{t}, \rho_{t+\delta t}\right)$ between neighboring states by

$$
d s^{2}=D_{B}^{2}\left(\rho_{t}, \rho_{t+d t}\right)=\frac{\mathcal{I}_{F}}{4} d t^{2},
$$

defining a metric in the space of density operators [91]. The Bures distance between any two states $\rho_{1}$ and $\rho_{2}$ is defined by $D_{B}\left(\rho_{1}, \rho_{2}\right):=\sqrt{2} \sqrt{1-\sqrt{F\left(\rho_{1}, \rho_{2}\right)}}$, where $F\left(\rho_{1}, \rho_{2}\right):=$ $\left(\operatorname{Tr}\left(\sqrt{\sqrt{\rho_{2}} \rho_{1} \sqrt{\rho_{2}}}\right)\right)^{2}$ is the Uhlmann fidelity. This connection has been exploited in the literature to derive a lower bound on the time needed for a system to evolve between orthogonal states $[18,94]$. 


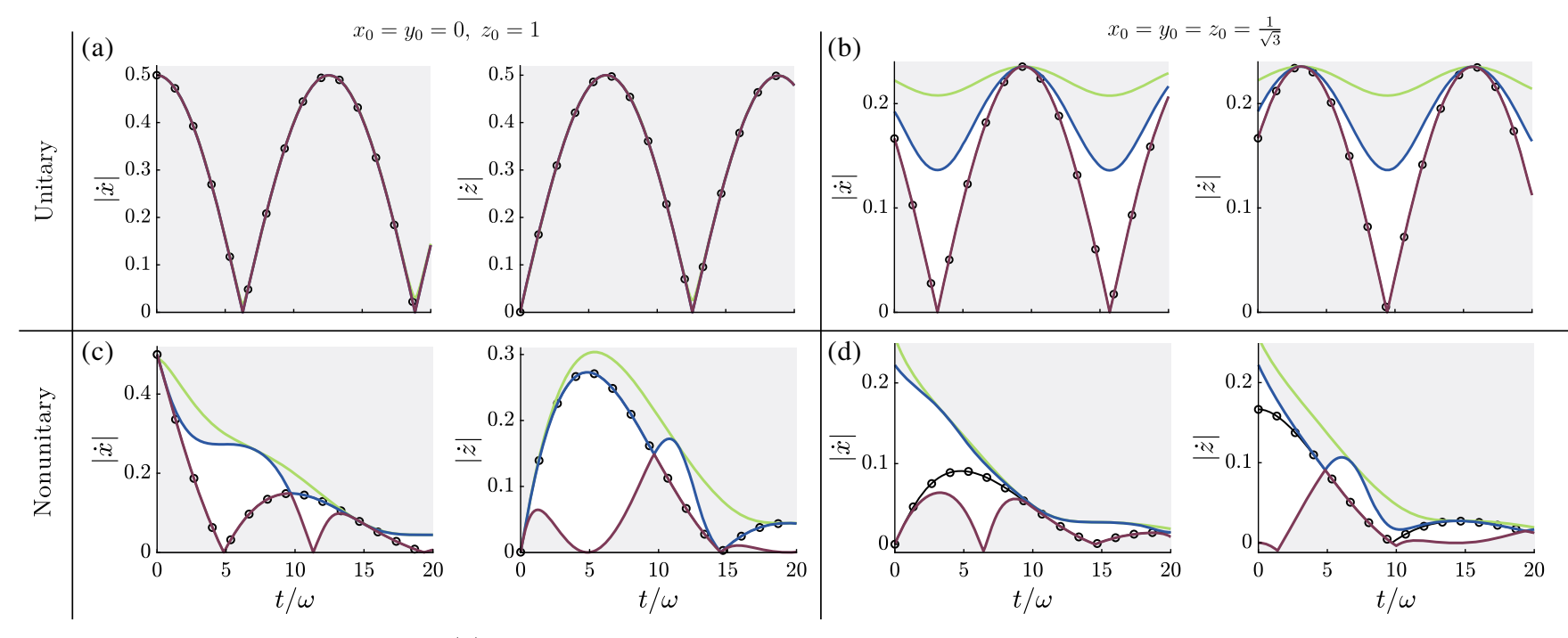

$\rightarrow$ Speed — Speed limit (3) — Coherent-incoherent speed limit — Coherent-incoherent lower speed limit

FIG. 1. Speed limits for observables on a qubit. The speed limit (3), derivable from the Cramér-Rao bound, and the coherentincoherent speed limits (12) and (11) impose constraints on the rate $\dot{a}$ at which observables evolve. We illustrate this on a qubit with a state parametrized by $\rho=\frac{1}{2}\left(\mathbb{1}+x \sigma_{x}+y \sigma_{y}+z \sigma_{z}\right)$ and driven by the Hamiltonian $H=(\omega / 2) \sigma_{y}$ and dephasing along $\sigma_{z}$ with a rate $\kappa$. Black lines with circles denote the speeds $|\dot{x}|$ and $|\dot{z}|$ of observables $\sigma_{x}$ and $\sigma_{z}$, and blue (red) lines denote the coherent-incoherent (lower) speed limits. The grayed areas denote rates forbidden by the coherent-incoherent speed limits. In green, the looser speed limit (3) appears inside the forbidden region. (a,c) The qubit is initialized in state $z_{0}=1$ and thus evolves in the $y=0$ plane on and inside the Bloch sphere, which in turn implies that both observables $\sigma_{z}$ and $\sigma_{x}$ have coherent-incoherent decompositions that satisfy conditions for the saturation of Eq. (10), with $\sigma_{\{z, x\}}=\alpha_{C}^{\{z, x\}} L_{C}+\alpha_{I}^{\{z, x\}} L_{I}$. For unitary dynamics $[\kappa=0$, (a)], this also means that the speed limit (3) saturates, and the three curves coincide. For open-system dynamics $[\kappa \neq 0,(\mathrm{c})]$, the red and black curves coincide, but the speed limit (3) is looser except when $\alpha_{C}^{\{z, x\}}=\alpha_{I}^{\{z, x\}}$. Alternatively, whenever $\alpha_{C}^{\{z, x\}}$ and $\alpha_{I}^{\{z, x\}}$ have the same (different) sign, the coherent-incoherent (lower) speed limits saturate. (b,d) The coherent-incoherent speed limits (12) and (11) are not tight for a qubit initialized in $x_{0}=y_{0}=z_{0}=1 / \sqrt{3}$, when the state does not evolve within the $y=0$ plane, and the observables no longer have a decomposition solely in terms of $L_{C}$ and $L_{I}$. Nevertheless, these bounds serve to constrain observables' dynamics more than the speed limit (3).

Equations (3) and (18) lead to an integrated bound that relates the change in the observable to the Bures length $\mathcal{L}\left(\rho_{i}, \rho_{f}\right)$ of the path followed by the system:

$\int_{0}^{\tau} \frac{|\dot{a}|}{\Delta A} d t \leq \int_{0}^{\tau} \sqrt{\mathcal{I}_{F}} d t=2 \int_{\rho_{i}}^{\rho_{f}} d s=2 \mathcal{L}\left(\rho_{i}, \rho_{f}\right)$

This new bound shows how the path taken by the system in state space puts constraints on the corresponding evolution of system observables. In particular, note that $A$ is not the Hamiltonian or the generator of evolution but an arbitrary observable of interest. The geodesic is the path that minimizes $\mathcal{L}\left(\rho_{i}, \rho_{f}\right)$, and its length is given by the Bures angle length $\mathcal{L}_{\min }\left(\rho_{i}, \rho_{f}\right)=\arccos \left[\sqrt{F\left(\rho_{i}, \rho_{f}\right)}\right]$ [10,91]. Figure 2 illustrates the constraints that Eq. (19) imposes on different paths taken by the system in state space.

Along the lines similar to the previous integrated bound, the change of an observable due to the change in the state from $\rho_{i}$ to $\rho_{f}$ over a time $\tau$ satisfies (Appendix H)

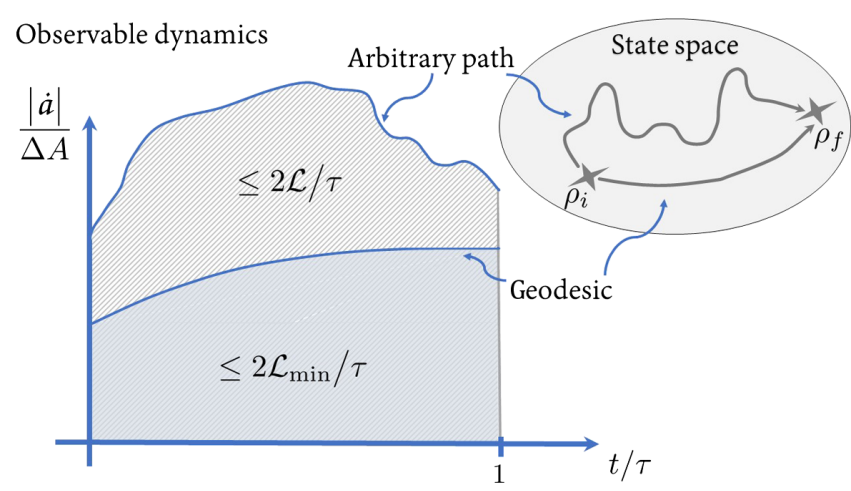

FIG. 2. Constraints on evolution of observables. The integrated speed limits (19) and (20) place constraints on the rate of change of an observable, which depend on the path taken by the state of the system in state space. The former bound says that the area under the curve $|\dot{a}| / \Delta A$ of an arbitrary path is upper bounded by the path length $\mathcal{L}$, as $\int|\dot{a}| / \Delta A d t \leq 2 \mathcal{L}$. Geodesics put a more stringent constraint on the dynamics of an observable: The area under the curve $|\dot{a}| / \Delta A$ must satisfy $\int|\dot{a}| / \Delta A d t \leq 2 \mathcal{L}_{\text {min }}$. Note that $A$ need not be related to the Hamiltonian or the generator of the dynamics but is rather an arbitrary observable of interest. 


$$
\begin{aligned}
|a(\tau)| & =\left|\int_{0}^{\tau} \dot{a} d t\right| \leq 2 \int_{\rho_{i}}^{\rho_{f}} \Delta A d s \\
& \leq 2 \sqrt{\mathcal{J}\left(\rho_{i}, \rho_{f}\right) \frac{1}{\tau} \int_{0}^{\tau}(\Delta A)^{2} d t .}
\end{aligned}
$$

The total change is thus bounded by the integrated fluctuations of the observable over the path through Hilbert space. This, in turn, is bounded by the integrated observable fluctuations and the divergence of the path $\mathcal{J}\left(\rho_{i}, \rho_{f}\right):=\tau \int_{0}^{\tau} \mathcal{I}_{F} d t$ in state space $[69,92,93]$.

The decomposition of dynamics into incoherent and coherent terms also provides a way to quantify the integrated contribution of each of them to the change in an observable. Often, quantum phenomena necessitate unitary dynamics in order to benefit from quantum mechanical advantages. For example, unavoidable incoherent effects stemming from experimental imperfections in isolating, preparing, or driving a system amount to errors in the resulting dynamics, hindering quantum cryptographic protocols [95] and computing [96-98] or destroying quantum correlations [99-101]. Integrating bound (10) on the incoherent contribution to the change of an observable gives a bound on how much the incoherent dynamics affects an observable:

$$
\begin{aligned}
\left|a(\tau)-a_{C}(\tau)\right|=\left|a_{I}(\tau)\right| & =\left|\int_{0}^{\tau} \operatorname{cov}\left(A_{I}, L_{I}\right) d t\right| \\
& \leq \int_{0}^{\tau} \Delta A_{I} \sqrt{\mathcal{I}_{F}^{I}} d t .
\end{aligned}
$$

This bound can be interpreted as quantifying the error that incoherent dynamics induces to the desired change $a_{C}(\tau)$ in an observable $A$, had the system evolved according to an ideal unitary evolution. An observable that cannot discriminate eigenstates of $\rho_{t}$ along its evolution, in the sense of $\langle j|A| j\rangle=\langle k|A| k\rangle \forall j, k$, obeys $\Delta A_{I}=0$ and thus does not accumulate an error due to incoherent evolution.

Alternatively,

$$
\begin{aligned}
\left|a(\tau)-a_{I}(\tau)\right| & =\left|\int_{0}^{\tau} \operatorname{cov}\left(A_{C}, L_{C}\right) d t\right| \\
& \leq \int_{0}^{\tau} \Delta A_{C} \sqrt{\mathcal{I}_{F}^{C}} d t
\end{aligned}
$$

bounds the maximum deviation that coherent dynamics can induce to an incoherent process driving an observable $A$. The looser bound $\left|a(\tau)-a_{I}(\tau)\right| \leq$ $2 \sqrt{\mathcal{J}_{C}\left(\rho_{i}, \rho_{f}\right)(1 / \tau) \int_{0}^{\tau}\left(\Delta A_{C}\right)^{2} d t}$ holds in terms of the quantum divergence $\mathcal{J}_{C}\left(\rho_{i}, \rho_{f}\right):=\tau \int_{0}^{\tau} \mathcal{I}_{F}^{C} d t$ of the path.

The maximum coherent speedup at any given time occurs when the coherent speed limit is saturated, e.g., when $L_{C} \propto A_{C}$ (see Sec. V). Using Eq. (8a), we prove in Appendix I that the Hamiltonian

$$
H_{t}^{\text {speedup }}:=-\lambda_{t} \frac{i}{2} \sum_{j \neq k}^{d} \frac{\left(p_{j}+p_{k}\right)}{p_{j}-p_{k}} A_{j k}|j\rangle\langle k|
$$

drives observable $A$ at such a speed limit. Here, the prefactor $\lambda_{t}$ sets the energy scale and would typically be determined by the available resources. The Hamiltonian $H_{t}^{\text {speedup }}=H_{t}^{\text {speedup }}\left(\rho_{t}, A\right)$ is tailored to the state of the system and the observable of interest, and makes optimal use of the energetic resources to coherently drive the observable at its speed limit and, as a result, change it by a value $\left|a_{C}(\tau)\right|=\int_{0}^{\tau} \Delta A_{C} \sqrt{\mathcal{I}_{F}^{C}} d t=\int_{0}^{\tau}\left(\mathcal{I}_{F}^{C} / \lambda_{t}\right) d t$ within a time $\tau$.

In Appendix I, we illustrate how to exploit $H_{t}^{\text {speedup }}$ to enhance an incoherent process that erases information stored in a qubit. We consider a system initialized in state $\left|\Psi_{0}\right\rangle=a|0\rangle+b|1\rangle$, with $a$ and $b$ real, for simplicity, and assume that it is critical to hide from an adversary the fact that $z_{0}:=\left\langle\sigma_{z}\right\rangle(0)=a-b$ is not equal to zero. An incoherent dynamics, modeled by a Lindblad master equation $\dot{\rho}_{t}=\gamma \sum_{l=0,1}\left(L_{l} \rho_{t} L_{l}^{\dagger}-\frac{1}{2}\left\{L_{l}^{\dagger} L_{l}, \rho_{t}\right\}\right)$ with jump operators $L_{0}=|r\rangle\langle 1|$ and $L_{1}=|r\rangle\langle 0|$, drives the system to an orthogonal state $|r\rangle$ at a rate $\gamma$. Since $\left\langle r\left|\sigma_{z}\right| r\right\rangle=0$, this dynamics incoherently erases $z_{0}$ at a rate $\gamma$. We find that the optimal Hamiltonian (23) that coherently enhances such a process is given by $H_{t}^{\text {speedup }}=\epsilon\left[\operatorname{sign}\left(z_{t}\right) / \operatorname{sign}\left(x_{t}\right)\right] \sigma_{y}$, where $\epsilon:=\left\|H_{t}^{\text {speedup }}\right\|$ is set by the available energetic resources. While the purely incoherent dynamics hides the fact that $z_{0} \neq 0$ at a rate $-\dot{z}_{t}^{\text {incoh }} / z_{t}^{\text {incoh }}=\gamma$, the unitarily enhanced process leads to a faster rate of $-\dot{z}_{t} / z_{t}=$ $\gamma+2 \epsilon\left|x_{t} / z_{t}\right|$.

In this way, the new speed limits (10), (12), and (11), and the bounds (21) and (22), pave the way to a systematic study of quantum speedups by identifying speed limits due to (i) incoherent dynamics, (ii) unitary quantum dynamics, and (iii) arbitrary quantum dynamics, and in doing so, to a better understanding of the regimes in which enhancements due to coherent dynamics occur.

\section{CONCLUSIONS}

We derived speed limits on expectation values of observables for a quantum system evolving under arbitrary differentiable dynamics. These bounds distinguish between classes of observables $A_{Q}$ driven solely by the Hamiltonian of a system and classes of observables $A_{C}$ driven solely by incoherent dynamics. An observable will typically have coherent and incoherent contributions, $A=A_{C}+A_{I}$, and its speed will be bounded by a linear combination of the coherent Fisher information $\mathcal{I}_{F}^{C}$ and the incoherent Fisher information $\mathcal{I}_{F}^{I}$, weighted by associated fluctuations in the contributions to the observable.

This division of dynamics in terms of coherent and incoherent contributions was crucial to deriving upper bounds on speed that are tighter than those implied by 
the quantum Cramér-Rao bound, where we exploited the fact that the quantum Cramér-Rao bound is loose when applied to a particular (nonoptimized) estimator. Our framework also allowed us to (i) prove lower bounds on the speed of evolution, (ii) quantify the effects from nonunitary open dynamics of a quantum system, and (iii) quantify the speedups that coherent dynamics can provide to incoherent processes.

We expect these advances to broaden the field of applications of quantum speed limits by better capturing the timescales that are involved in the dynamics of different physical system observables. Our work also takes a step towards speed limits that correctly capture the dynamics of many-body systems, where it is known that speed limits are largely loose in estimating relevant timescales, e.g., for thermalization of quantum systems [102,103]. A particularly interesting avenue for future research is that of incorporating constraints on the dynamics of physical systems, such as locality [104], integrability [105], or those imposed by limited controllability [106-109].

\section{ACKNOWLEDGMENTS}

L. P. G. P. is thankful for extensive feedback from Jake Bringewatt, and for interesting discussions with Igor Boettcher, Lucas Brady, Stefano Cusumano, Yi-Kai Liu, Łukasz Rudnicki, and Oles Shtanko. Some authors of this work were supported by the AFOSR MURI project "Scalable Certification of Quantum Computing Devices and Networks," the U.S. Department of Energy (DOE) ASCR Quantum Testbed Pathfinder program (Award No. DE-SC0019040), the DOE ASCR Accelerated Research in Quantum Computing program (Award No. DE-SC0020312), U.S. DOE Award No. DESC0019449, the NSF PFCQC program, AFOSR, ARO MURI, AFOSR MURI, PID2019-109007GA-I00, the John Templeton Foundation, the Spanish Ministerio de Ciencia e Innovación (PID2019-109007GA-I00), and the National Science Foundation under Grant No. 1856250.

\section{APPENDIX A: EQUATION OF MOTION FOR THE EXPECTATION VALUE OF OBSERVABLES}

In this appendix, we derive Eq. (2) of Sec. II in the main text; i.e., we show that the change in the expectation value of an observable is characterized by the covariance between the observable and the symmetric logarithmic derivative.

Since the symmetric logarithmic derivative $L$ is implicitly defined by the expression $\left(d \rho_{t} / d t\right)=: \frac{1}{2}\left\{\rho_{t}, L\right\}$, where $\{A, B\}=A B+B A$ denotes an anticommutator, we see that the change in an observable due to changes in the state satisfies

$$
\begin{aligned}
\operatorname{Tr}\left(\frac{d \rho_{t}}{d t} A\right) & =\operatorname{Tr}\left(\frac{1}{2}\left\{\rho_{t}, L\right\} A\right) \\
& =\frac{1}{2} \operatorname{Tr}\left(\rho_{t}\{L, A\}\right) \\
& =\frac{1}{2} \operatorname{Tr}\left(\rho_{t}\{L, A\}\right)-\langle L\rangle\langle A\rangle \\
& =\operatorname{cov}(L, A)=: \dot{a},
\end{aligned}
$$

where we use the facts that the trace is cyclic and that $\langle L\rangle=\operatorname{Tr}\left(\rho_{t} L\right)=\frac{1}{2} \operatorname{Tr}\left(\left\{\rho_{t}, L\right\}\right)=\operatorname{Tr}\left(d \rho_{t} / d t\right)=0$ for tracepreserving dynamics. Here, $\operatorname{cov}(A, L):=\frac{1}{2} \operatorname{Tr}\left(\rho_{t}\{A, L\}\right)-$ $\langle A\rangle\langle L\rangle$ is the symmetrized covariance between operators $L$ and $A$.

The expectation value $\langle A\rangle=\operatorname{Tr}\left(A \rho_{t}\right)$ thus follows the equation of motion

$$
\begin{aligned}
\frac{d\langle A\rangle}{d t} & =\operatorname{Tr}\left(\frac{d \rho_{t}}{d t} A\right)+\left\langle\frac{d A}{d t}\right\rangle \\
& =\operatorname{cov}(L, A)+\left\langle\frac{d A}{d t}\right\rangle=: \dot{a}+\left\langle\frac{d A}{d t}\right\rangle,
\end{aligned}
$$

which is Eq. (2) of the main text.

\section{APPENDIX B: ASSUMPTIONS ON THE SUPPORT OF THE STATE}

In this appendix, we study the effect that a change in the rank of the state has on the speed of an observable. Specifically, we show that bound (3) in the main text is exact for states with constant support, and we obtain an estimate of the error to the bound in cases when the rank of the state changes and the incoherent Fisher information diverges.

In deriving the first general bound [Eq. (3) in the main text]

$$
|\dot{a}| \leq \Delta A \sqrt{\mathcal{I}_{F}},
$$

we disregard the contribution of levels $j$ such that $p_{j}=0$. Here, we quantify the error introduced by this and argue that such an assumption gives a good approximation for most times for differentiable continuous evolution.

Defining $\delta A:=A-\langle A\rangle$, we find

$$
\begin{aligned}
|\dot{a}| & =\left|\operatorname{Tr}\left(\delta A \frac{d \rho_{t}}{d t}\right)\right|=\left|\sum_{j k} \delta A_{j k}\left\langle k\left|\frac{d \rho_{t}}{d t}\right| j\right\rangle\right| \\
& =\left|\sum_{p_{j}, p_{k} \neq 0} \delta A_{j k}\left\langle k\left|\frac{d \rho_{t}}{d t}\right| j\right\rangle+\sum_{p_{j}, p_{k}=0} \delta A_{j k}\left\langle k\left|\frac{d \rho_{t}}{d t}\right| j\right\rangle\right| \\
& \leq\left|\sum_{p_{j}, p_{k} \neq 0} \delta A_{j k}\left\langle k\left|\frac{d \rho_{t}}{d t}\right| j\right\rangle\right|+\left|\sum_{p_{j}, p_{k}=0} \delta A_{j k}\left\langle k\left|\frac{d \rho_{t}}{d t}\right| j\right\rangle\right|,
\end{aligned}
$$


where we set aside the sum of terms with $p_{j}=0$ and denote matrix elements by $\langle k|\delta A| j\rangle=\delta A_{j k}$. The first term includes all contributions to the rate of change that satisfy $p_{j} \neq 0$, as assumed in the main text, giving rise to Eq. (B1), which is also Eq. (3) in the main text. The second term thus contains all factors that are disregarded in the bound $|\dot{a}| \leq$ $\Delta A \sqrt{\mathcal{I}_{F}}$ when states with $p_{j}=0$ exist.

For the second term, using the fact that any tracepreserving differentiable evolution can be expressed as

$$
\frac{d}{d t} \rho_{t}=-i\left[H_{t}, \rho_{t}\right]+U_{t} \frac{d \chi_{t}}{d t} U_{t}^{\dagger},
$$

and that $|j\rangle=U_{t}|j\rangle_{0}$ and $\chi_{t}=\sum_{j} p_{j}|j\rangle_{00}\langle j|$, we find that

$$
\begin{aligned}
\sum_{p_{j}, p_{k}=0} \delta A_{j k}\left\langle k\left|\frac{d \rho_{t}}{d t}\right| j\right\rangle & =\sum_{p_{j}, p_{k}=0} \delta A_{j k}\left\langle k\left|U_{t} \frac{d \chi_{t}}{d t} U_{t}^{\dagger}\right| j\right\rangle \\
& =\sum_{p_{j}, p_{k}=0} \delta A_{j k}\left\langle k\left|U_{t} \sum_{l} \dot{p}_{l}\right| l\right\rangle_{00}\left\langle l\left|U_{t}^{\dagger}\right| j\right\rangle \\
& =\sum_{p_{j}, p_{k}=0} \sum_{l} \dot{p}_{l} \delta A_{j k}\langle k \mid l\rangle\langle l \mid j\rangle \\
& =\sum_{p_{j}=0} \dot{p}_{j} \delta A_{j j}=\sum_{\left\{p_{j}=0\right\}} \dot{p}_{j} A_{j j}
\end{aligned}
$$

This term, which would result in a correction to Eq. (10b) in the main text, contributes very little to the rate of change of $\dot{a}$ for continuous evolutions because it is nonzero only for infinitesimal moments in time in which a nonpopulated state $j$ acquires a nonzero probability $p_{j}$. Whenever a state has constant support, the error term is zero.

\section{APPENDIX C: COHERENT AND INCOHERENT CONTRIBUTIONS TO SPEED LIMITS ON OBSERVABLES}

In this appendix, we derive speed limits on the coherent and incoherent contributions of an observable, proving Eqs. (10a), (10b), and (12) of Sec. III in the main text.

The dynamics of any quantum system can be decomposed in terms of coherent and incoherent contributions as

$$
\frac{d}{d t} \rho_{t}=-i\left[H_{t}, \rho_{t}\right]+U_{t} \frac{d \chi_{t}}{d t} U_{t}^{\dagger}
$$

where the Hamiltonian $H_{t}:=i\left(d U_{t} / d t\right) U_{t}^{\dagger}$ drives the coherent dynamics and where $\chi_{t}=\sum_{j} p_{j}(t)|j\rangle_{00}\langle j|$, the eigenvalues of $\rho_{t}=\sum_{j} p_{j}|j\rangle\langle j|$ are $\left\{p_{j}(t)\right\}$, and $|j\rangle=U_{t}|j\rangle_{0}$.

Similarly, different components of an observable will evolve under the coherent and incoherent contributions to the dynamics. We define a coherent-incoherent separation of the observable of interest $A:=A_{C}+A_{I}$, with

$$
A_{C}:=\sum_{j \neq k} A_{j k}|j\rangle\left\langle k\left|, \quad A_{I}:=\sum_{j} A_{j j}\right| j\right\rangle\langle j| .
$$

The change $\dot{a}_{C}:=\operatorname{Tr}\left[\left(d \rho_{t} / d t\right) A_{C}\right]$ of the observable's coherent component is

$$
\begin{aligned}
\dot{a}_{C} & =\sum_{j \neq k} A_{j k}\left\langle k\left|\frac{d \rho_{t}}{d t}\right| j\right\rangle=-i \sum_{j \neq k} A_{j k}\left\langle k\left|\left[H_{t}, \rho_{t}\right]\right| j\right\rangle+\sum_{j \neq k} A_{j k}\left\langle k\left|U_{t} \frac{d \chi_{t}}{d t} U_{t}^{\dagger}\right| j\right\rangle \\
& =-i \sum_{j \neq k} A_{j k} \frac{\left\langle k\left|\left[H_{t}, \rho_{t}\right]\right| j\right\rangle}{p_{j}+p_{k}}\left(p_{j}+p_{k}\right)+\sum_{j \neq k} A_{j k 0}\left\langle k\left|\left(\sum_{l} \dot{p}_{l}(t)|l\rangle_{00}\langle l|\right)\right| j\right\rangle_{0} \\
& =\frac{1}{2} \operatorname{Tr}\left(A_{C} L_{C} \rho_{t}\right)+\frac{1}{2} \operatorname{Tr}\left(A_{C} \rho_{t} L_{C}\right)+\sum_{j \neq k} A_{j k} \dot{p}_{j}(t) \delta_{j k} \\
& =\operatorname{cov}\left(A_{C}, L_{C}\right)
\end{aligned}
$$

where we used the fact that the diagonal components $A_{j j}$ of the coherent component $A_{C}$ of the observable are zero, and that conservation of probability gives $\left\langle L_{C}\right\rangle=0$, where

$$
\begin{aligned}
L_{C} & :=-2 i \sum_{j \neq k} \frac{\left\langle j\left|\left[H_{t}, \rho_{t}\right]\right| k\right\rangle}{\left(p_{j}+p_{k}\right)}|j\rangle\langle k| \\
& =-2 i \sum_{j \neq k} \frac{\left\langle k\left|\left[H_{t}, \rho_{t}\right]\right| j\right\rangle}{\left(p_{j}+p_{k}\right)}|k\rangle\langle j| .
\end{aligned}
$$

The speed limit follows from the Cauchy-Schwarz inequality:

$$
\begin{aligned}
\left|\dot{a}_{C}\right|= & \left|\operatorname{cov}\left(A_{C}, L_{C}\right)\right|=\frac{1}{2}\left|\operatorname{Tr}\left(\rho_{t} \delta A_{C} \delta L_{C}\right)+\operatorname{Tr}\left(\rho_{t} \delta L_{C} \delta A_{C}\right)\right| \\
\leq & \frac{1}{2}\left\{\sqrt{\operatorname{Tr}\left[\rho_{t}\left(\delta A_{C}\right)^{2}\right] \operatorname{Tr}\left[\rho_{t}\left(\delta L_{C}\right)^{2}\right]}\right. \\
& \left.+\sqrt{\operatorname{Tr}\left[\rho_{t}\left(\delta A_{C}\right)^{2}\right] \operatorname{Tr}\left[\rho_{t}\left(\delta L_{C}\right)^{2}\right]}\right\}=\Delta A \Delta L_{C}, \quad \text { (C5) }
\end{aligned}
$$

where we define $\delta A_{C}:=A_{C}-\left\langle A_{C}\right\rangle$ and $\delta L_{C}:=L_{C}-\left\langle L_{C}\right\rangle$.

A direct calculation further shows that

$$
\Delta L_{C}=\operatorname{Tr}\left(\rho_{t} L_{C}^{2}\right)=\mathcal{I}_{F}^{C}=2 \sum_{j \neq k} \frac{\left|\left\langle j\left|\left[H_{t}, \rho_{t}\right]\right| k\right\rangle\right|^{2}}{p_{j}+p_{k}}
$$


is the coherent contribution to the quantum Fisher information.

This then proves Eq. (10a) in the main text:

$$
\left|\dot{a}_{C}\right|=\left|\operatorname{cov}\left(A, L_{C}\right)\right| \leq \Delta A \Delta L_{C}=\Delta A_{C} \sqrt{\mathcal{I}_{F}^{C}} .
$$

For the evolution of the incoherent part, we use the fact that $A_{I}$ is diagonal in the basis of $\rho_{t}$, so that

$$
\begin{aligned}
\dot{a}_{I} & =\sum_{j k} \delta A_{j j}\left\langle k\left|\frac{d \rho_{t}}{d t}\right| j\right\rangle=\sum_{j} \delta A_{j j}\left\langle j\left|U_{t} \frac{d \chi_{t}}{d t} U_{t}^{\dagger}\right| j\right\rangle \\
& =\sum_{j} \delta A_{j j 0}\left\langle j\left|\left(\sum_{l} \dot{p}_{l}(t)|l\rangle_{00}\langle l|\right)\right| j\right\rangle_{0}=\sum_{j} \delta A_{j j} \dot{p}_{j}(t) .
\end{aligned}
$$

This becomes identical to the expression for the change in a classical observable acting on a classical stochastic system. It was shown in Ref. [50] that the dynamics of classical observables under stochastic dynamics satisfies a speed limit that depends on the classical Fisher information $\mathcal{I}_{F}^{I}:=\sum_{j} p_{j}\left[(d / d t) \ln p_{j}\right]^{2}$.

Defining

$$
L_{I}:=\sum_{j} \frac{d \ln p_{j}}{d t}|j\rangle\langle j|
$$

and disregarding states with $p_{j}=0$ (see Appendix B for an expression of the error introduced by this), we get

$$
\begin{aligned}
\dot{a}_{I} & =\sum_{j} \dot{p}_{j}(t) A_{j j}=\sum_{j} p_{j} \frac{\dot{p}_{j}}{p_{j}} A_{j j}=\sum_{j} p_{j} \frac{d}{d t} \ln \left(p_{j}\right) A_{j j} \\
& =\operatorname{Tr}\left(\rho_{t} L_{I} A\right)=\operatorname{cov}\left(A_{I}, L_{I}\right),
\end{aligned}
$$

where we used the fact that $\left\langle L_{I}\right\rangle=0$. The Cauchy-Schwarz inequality then gives

$$
\left|\dot{a}_{I}\right|=\left|\operatorname{cov}\left(A_{I}, L_{I}\right)\right| \leq \Delta A_{I} \Delta L_{I}=\Delta A_{I} \sqrt{\mathcal{I}_{F}^{I}},
$$

which proves Eq. (10b) in the main text.

Combining the results gives

$$
|\dot{a}| \leq\left|\dot{a}_{C}\right|+\left|\dot{a}_{I}\right| \leq \Delta A_{C} \sqrt{\mathcal{I}_{F}^{C}}+\Delta A_{I} \sqrt{\mathcal{I}_{F}^{I}}
$$

proving the coherent-incoherent decomposition of the central bound in the main text, Eq. (12).

The reverse triangle inequality [81] implies that $|x+y| \geq|x|-|y|$. Combining this with the fact that $\dot{a}=$ $\dot{a}_{C}+\dot{a}_{I}$ and with bounds (C7) and (C12) gives

$$
\begin{aligned}
& |\dot{a}|=\left|\dot{a}_{C}+\dot{a}_{I}\right| \geq\left|\dot{a}_{C}\right|-\left|\dot{a}_{I}\right| \geq\left|\dot{a}_{C}\right|-\Delta A_{I} \sqrt{\mathcal{I}_{F}^{I}}, \\
& |\dot{a}|=\left|\dot{a}_{C}+\dot{a}_{I}\right| \geq\left|\dot{a}_{I}\right|-\left|\dot{a}_{C}\right| \geq\left|\dot{a}_{I}\right|-\Delta A_{C} \sqrt{\mathcal{I}_{F}^{C}} .
\end{aligned}
$$

This proves the lower speed limit in Eq. (11) of the main text.

Finally, we note that the quantum Fisher information defined by Eq. (4) in the main text can be decomposed in terms of the coherent and incoherent contributions to the dynamics, $\mathcal{I}_{F}=\mathcal{I}_{F}^{C}+\mathcal{I}_{F}^{I}$, with

$$
\begin{aligned}
& \mathcal{I}_{F}^{C}:=\Delta L_{C}=2 \sum_{j \neq k} \frac{\left|\left\langle j\left|\left[H_{t}, \rho_{t}\right]\right| k\right\rangle\right|^{2}}{p_{j}+p_{k}}, \\
& \mathcal{I}_{F}^{I}:=\Delta L_{I}=\sum_{j} p_{j}\left(\frac{d}{d t} \ln p_{j}\right)^{2} .
\end{aligned}
$$

\section{APPENDIX D: UPPER BOUND ON THE INCOHERENT FISHER INFORMATION}

In this appendix, we derive an upper bound on the incoherent Fisher information for a system interacting with an environment. We prove the second part of Eq. (14) in Sec. IV of the main text, and we prove that the bound is loose.

The quantum Fisher information is $\mathcal{I}_{F}=\mathcal{I}_{F}^{C}+\mathcal{I}_{F}^{I}$, where

$\mathcal{I}_{F}^{C}:=2 \sum_{j \neq k} \frac{\left|\left\langle j\left|\left[H_{t}, \rho_{t}\right]\right| k\right\rangle\right|^{2}}{p_{j}+p_{k}}, \quad \mathcal{I}_{F}^{I}:=\sum_{j} p_{j}\left(\frac{d}{d t} \ln p_{j}\right)^{2}$.

Braunstein and Caves proved an upper bound to the coherent Fisher information,

$$
\mathcal{I}_{F}^{C} \leq 4\left(\Delta H_{t}\right)^{2},
$$

where $H_{t}$ is the Hamiltonian of the system [53]. Equality holds only if the state is pure or in the trivial case $H_{t} \propto \mathbb{1}$.

Here, we prove an analogous bound for the incoherent Fisher information,

$$
\mathcal{I}_{F}^{I}=\sum_{j} p_{j}\left(\frac{d}{d t} \ln p_{j}\right)^{2}=\sum_{j} \frac{\left(\dot{p}_{j}\right)^{2}}{p_{j}} .
$$

Let us assume that the incoherent dynamics stems from the interaction between the system and a second system, i.e., an environment. The two interact via a Hamiltonian $H_{t}^{\text {int }}$. Then, if $H_{t}$ and $H_{t}^{\mathcal{E}}$ represent the Hamiltonians of the system of interest and the environment, the total systemenvironment Hamiltonian is $H_{t}^{\mathcal{S}}=H_{t} \otimes \mathbb{1}_{\mathcal{E}}+\mathbb{1}_{\mathcal{S}} \otimes$ $H_{t}^{\mathcal{E}}+H_{t}^{\text {int }}$. Note that self-Hamiltonians $H_{t}$ and $H_{t}^{\mathcal{E}}$ do not change the eigenvalues of the state of the system, so $H_{t}^{\text {int }}$ is the only source of $\mathcal{I}_{F}^{I} \neq 0$. We assume that $H_{t}^{\text {int }}$ has support on the Hilbert space of the system and the environment and that it acts on the state causing incoherent dynamics of the system. This excludes, for instance, cases in which the system or the environment are in eigenstates of $H_{t}^{\text {int }}$. 
Then, we have

$$
\begin{aligned}
\dot{p}_{j} & =\left\langle j\left|\frac{d \rho_{t}}{d t}\right| j\right\rangle=\left\langle j\left|-i\left[H_{t}, \rho_{t}\right]-i \operatorname{Tr}_{\mathcal{E}}\left(\left[H_{t}^{\mathrm{int}}, \rho_{t}^{\mathcal{S E}}\right]\right)\right| j\right\rangle \\
& =-i \sum_{e}\left\langle j\left|\left\langle e\left|\left[H_{t}^{\mathrm{int}}, \rho_{t}^{\mathcal{S E}}\right]\right| e\right\rangle\right| j\right\rangle,
\end{aligned}
$$

where $\rho_{t}^{\mathcal{S E}}$ is the joint state of the system $\mathcal{S}$ and the environment $\mathcal{E}, \rho_{t}:=\operatorname{Tr}_{\mathcal{E}}\left(\rho_{t}^{\mathcal{S E}}\right)=\sum_{e}\left\langle e\left|\rho_{t}^{\mathcal{S E}}\right| e\right\rangle$ is the state of the system, and $\{|e\rangle\}$ denotes an arbitrary basis in the Hilbert space of $\mathcal{E}$. Defining the projector $X_{j}:=$ $\sum_{e}|e\rangle|j\rangle\langle j|\langle e|$ and the shifted Hamiltonian $\delta H_{t}^{\mathrm{int}}:=$ $H_{t}^{\text {int }}-\operatorname{Tr}\left(H_{t}^{\text {int }} \rho_{t}^{\mathcal{S E}}\right)$, we get

$$
\begin{aligned}
\mathcal{I}_{F}^{I} & =\sum_{j} \frac{\left(\dot{p}_{j}\right)^{2}}{p_{j}}=\sum_{j} \frac{\left|\sum_{e}\left\langle j\left|\left\langle e\left|\left[H_{t}^{\mathrm{int}}, \rho_{t}^{\mathcal{S E}}\right]\right| e\right\rangle\right| j\right\rangle\right|^{2}}{p_{j}}=\sum_{j} \frac{\left|\operatorname{Tr}\left(X_{j}\left[\delta H_{t}^{\mathrm{int}}, \rho_{t}^{\mathcal{S E}}\right]\right)\right|^{2}}{p_{j}} \\
& \leq \sum_{j} \frac{\left|2 \operatorname{Tr}\left(X_{j} \delta H_{t}^{\mathrm{int}} \rho_{t}^{\mathcal{S E}}\right)\right|^{2}}{p_{j}}=4 \sum_{j} \frac{\left|\operatorname{Tr}\left(X_{j} \delta H_{t}^{\mathrm{int}} \sqrt{\rho_{t}^{\mathcal{S E}}} \sqrt{\rho_{t}^{\mathcal{S E}}} X_{j}\right)\right|^{2}}{p_{j}} \\
& \leq 4 \sum_{j} \frac{\operatorname{Tr}\left(X_{j} \delta H_{t}^{\mathrm{int}} \rho_{t}^{\mathcal{S E}} \delta H_{t}^{\mathrm{int}} X_{j}\right) \operatorname{Tr}\left(X_{j} \rho_{t}^{\mathcal{S E}} X_{j}\right)}{p_{j}}=4 \sum_{j} \frac{\operatorname{Tr}\left(X_{j} \delta H_{t}^{\mathrm{int}} \rho_{t}^{\mathcal{S E}} \delta H_{t}^{\mathrm{int}}\right) \operatorname{Tr}\left(X_{j} \rho_{t}^{\mathcal{S E}}\right)}{p_{j}} \\
& =4 \sum_{j} \frac{\operatorname{Tr}\left(\sum _ { e } | e \rangle | j \rangle \left\langlej\left|\langle e| \delta H_{t}^{\mathrm{int}} \rho_{t}^{\mathcal{S E}} \delta H_{t}^{\mathrm{int}}\right) \operatorname{Tr}\left(\sum_{e^{\prime}}\left|e^{\prime}\right\rangle|j\rangle\langle j|\left\langle e^{\prime}\right| \rho_{t}^{\mathcal{S E}}\right)\right.\right.}{p_{j}} \\
& =4 \sum_{j} \frac{\left\langle j\left|\operatorname{Tr}_{\mathcal{E}}\left(\delta H_{t}^{\mathrm{int}} \rho_{t}^{\mathcal{S E}} \delta H_{t}^{\mathrm{int}}\right)\right| j\right\rangle\left\langle j\left|\operatorname{Tr}_{\mathcal{E}}\left(\rho_{t}^{\mathcal{S E}}\right)\right| j\right\rangle}{p_{j}}=4 \sum_{j} \frac{\left\langle j\left|\operatorname{Tr}_{\mathcal{E}}\left(\delta H_{t}^{\mathrm{int}} \rho_{t}^{\mathcal{S E}} \delta H_{t}^{\mathrm{int}}\right)\right| j\right\rangle\left\langle j\left|\rho_{t}\right| j\right\rangle}{p_{j}} \\
& =4 \sum_{j}\left\langle j\left|\operatorname{Tr}_{\mathcal{E}}\left(\delta H_{t}^{\mathrm{int}} \rho_{t}^{\mathcal{S E}} \delta H_{t}^{\mathrm{int}}\right)\right| j\right\rangle=4 \operatorname{Tr}\left(\left(\delta H_{t}^{\mathrm{int}}\right)^{2} \rho_{t}^{\mathcal{S E}}\right)=4\left(\Delta H_{t}^{\mathrm{int}}\right)^{2} .
\end{aligned}
$$

We used the fact that $|\operatorname{Tr}(A B C)|=|\operatorname{Tr}(A C B)|$ holds for Hermitian operators in the third line. We also used the facts that $X_{j}$ is a projector and $\rho_{t}^{\mathcal{S E}}$ is positive to apply the Cauchy-Schwarz inequality on line four. The variance of the interaction Hamiltonian between the system and the environment is $\left(\Delta H_{t}^{\text {int }}\right)^{2}:=\operatorname{Tr}\left(\left(\delta H_{t}^{\text {int }}\right)^{2} \rho_{t}^{\mathcal{S E}}\right)$. Then, we get the second part of Eq. (14) in the main text:

$$
\mathcal{I}_{F}^{I} \leq 4\left(\Delta H_{t}^{\mathrm{int}}\right)^{2},
$$

mirroring the bound on the coherent Fisher information in terms of the variance of the system Hamiltonian, i.e., the first part of Eq. (14) in the main text.

The proof of Eq. (D5) involves the Cauchy-Schwarz inequality between the operators $V_{j}:=X_{j} \delta H_{t}^{\text {int }} \sqrt{\rho_{t}^{\mathcal{S E}}}$ and $W_{j}:=X_{j} \sqrt{\rho_{t}^{\mathcal{S E}}}$, which is tight if and only if $V_{j}=$ $\left[\operatorname{Tr}\left(V_{j} W_{j}^{\dagger}\right) / \operatorname{Tr}\left(W_{j} W_{j}^{\dagger}\right)\right] W_{j}$ or, in the trivial case, when one of the operators is null. We now prove that the former is never the case. We have that

$$
\begin{aligned}
\sum_{j} \operatorname{Tr}\left(V_{j} \sqrt{\rho_{t}^{\mathcal{S E}}}\right) & =\sum_{j} \operatorname{Tr}\left(X_{j} \delta H_{t}^{\mathrm{int}} \rho_{t}^{\mathcal{S E}}\right) \\
& =\operatorname{Tr}\left(\delta H_{t}^{\mathrm{int}} \rho_{t}^{\mathcal{S E}}\right)=0
\end{aligned}
$$

$$
\sum_{j} \operatorname{Tr}\left(W_{j} \sqrt{\rho_{t}^{\mathcal{S E}}}\right)=\sum_{j} \operatorname{Tr}\left(X_{j} \rho_{t}^{\mathcal{S E}}\right)=\operatorname{Tr}\left(\rho_{t}^{\mathcal{S E}}\right)=1,
$$

where we used $\delta H_{t}^{\text {int }}:=H_{t}^{\text {int }}-\operatorname{Tr}\left(H_{t}^{\text {int }} \rho_{t}^{\mathcal{S E}}\right)$. This proves that one cannot have $V_{j} \propto W_{j}$ and that the bound in Eq. (D5) is loose except in the trivial case when both sides of the inequality are null, as stated after Eq. (14) in Sec. IV of the main text.

\section{APPENDIX E: A COROLLARY-SMALL INCREMENTAL ENTANGLING THEOREM WITHOUT ANCILLAS}

In this appendix, we focus on speed limits for the von Neumann entropy, which result in a simple proof of the small incremental entangling theorem in the ancillafree case stated below Eq. (15) in the main text: $|\dot{S}| \lesssim \ln d\left\|H_{t}^{\mathrm{int}}\right\|$.

The von Neumann entropy can be expressed as the expectation value of the surprisal operator $\left[-\ln \rho_{t}\right]$, $S=\left\langle-\ln \rho_{t}\right\rangle=-\operatorname{Tr}\left(\rho_{t} \ln \rho_{t}\right)$. Following Refs. [83,84], the variance of the surprisal operator satisfies 


$$
\begin{aligned}
(\Delta S)^{2} & :=\operatorname{Tr}\left(\rho_{t}\left(\ln \rho_{t}\right)^{2}\right)-S^{2} \\
& =(\ln (2))^{2}\left[\operatorname{Tr}\left(\rho_{t}\left(\log \rho_{t}\right)^{2}\right)-\left(\operatorname{Tr}\left(\rho_{t} \log \rho_{t}\right)\right)^{2}\right] \\
& \leq(\ln (2))^{2}\left[\frac{(\log (d-1))^{2}}{4}+\frac{1}{(\ln (2))^{2}}\right] \\
& =\frac{(\ln (d-1))^{2}}{4}+1,
\end{aligned}
$$

where we used the fact that $\ln (x)=\ln (2) \log (x)$, with $\log (x):=\log _{2}(x)$. Combined with Eqs. (14) and (15), this gives

$$
|\dot{S}| \leq \Delta S \sqrt{\mathcal{I}_{F}^{I}} \leq \sqrt{(\ln (d-1))^{2}+4} \Delta H_{t}^{\mathrm{int}},
$$

where $d$ is the dimension of the Hilbert space of the system. In the limit $d \gg 1$, this is

$$
|\dot{S}| \lesssim \ln (d) \Delta H_{t}^{\mathrm{int}} \leq \ln (d)\left\|H_{t}^{\mathrm{int}}\right\|,
$$

where the spectral norm $\left\|H_{t}^{\text {int }}\right\|$ is given by the largest eigenvalue of $H_{t}^{\text {int }}$. The last inequality recovers the scaling of the small entangling theorem in the ancilla-free case $[85,86]$.

\section{APPENDIX F: COMPARISON OF THE COHERENT-INCOHERENT BOUND (12) AND BOUND (3)}

Here, we compare the novel bound (12) with bound (3), derivable from the Cramér-Rao inequality. We show that the former is tighter than the latter, and we prove Eq. (17) of Sec. V in the main text.
Equation (3) in the main text reads

$$
|\dot{a}| \leq \Delta A \sqrt{\mathcal{I}_{F}} .
$$

We now prove that this speed limit is looser than the coherent-incoherent upper bound [Eq. (12) in the main text], which reads

$$
|\dot{a}| \leq\left|\dot{a}_{C}\right|+\left|\dot{a}_{I}\right| \leq \Delta A_{C} \sqrt{\mathcal{I}_{F}^{C}}+\Delta A_{I} \sqrt{\mathcal{I}_{F}^{I}}
$$

where $\mathcal{I}_{F}=\mathcal{I}_{F}^{C}+\mathcal{I}_{F}^{I}$. To do this, we first note that $A=A_{C}+A_{I}$, with

$$
A_{C}:=\sum_{j \neq k} A_{j k}|j\rangle\left\langle k\left|, \quad A_{I}:=\sum_{j} A_{j j}\right| j\right\rangle\langle j|,
$$

which implies that $\operatorname{Tr}\left(\rho_{t} A\right)=\operatorname{Tr}\left(\rho_{t} A_{I}\right)$ and $\operatorname{Tr}\left(\rho_{t} A_{C}\right)=0$. Then,

$$
\begin{aligned}
(\Delta A)^{2} & =\operatorname{Tr}\left(\rho_{t}\left(A_{C}+A_{I}\right)^{2}\right)-\left[\operatorname{Tr}\left(\rho_{t}\left(A_{C}+A_{I}\right)\right)\right]^{2} \\
& =\operatorname{Tr}\left(\rho_{t} A_{C}^{2}\right)+\operatorname{Tr}\left(\rho_{t} A_{I}^{2}\right)+2 \operatorname{Tr}\left(\rho_{t} A_{C} A_{I}\right)-\left[\operatorname{Tr}\left(\rho_{t} A_{I}\right)\right]^{2} \\
& =\left(\Delta A_{C}\right)^{2}+\left(\Delta A_{I}\right)^{2}, \quad(\mathrm{~F} 4)
\end{aligned}
$$

where, in the last line, we used the fact that $\operatorname{Tr}\left(\rho_{t} A_{C} A_{I}\right)=$ $\sum_{j k} p_{j}\left(A_{C}\right)_{j k}\left(A_{I}\right)_{k j}=0$ since $A_{C}$ has zero diagonal elements while $A_{I}$ is nonzero only on the diagonal.

Dividing the right-hand side of Eq. (F1) by the righthand side of Eq. (F2) gives

$$
\begin{aligned}
& \frac{\Delta A \sqrt{\mathcal{I}_{F}}}{\Delta A_{C} \sqrt{\mathcal{I}_{F}^{C}}+\Delta A_{I} \sqrt{\mathcal{I}_{F}^{I}}}=\sqrt{\frac{\left[\left(\Delta A_{C}\right)^{2}+\left(\Delta A_{I}\right)^{2}\right]\left(\mathcal{I}_{F}^{C}+\mathcal{I}_{F}^{I}\right)}{\Delta A_{C} \sqrt{\mathcal{I}_{F}^{C}}+\Delta A_{I} \sqrt{\mathcal{I}_{F}^{I}}}}=\sqrt{\frac{\left[\left(\Delta A_{C}\right)^{2}+\left(\Delta A_{I}\right)^{2}\right]\left(\mathcal{I}_{F}^{C}+\mathcal{I}_{F}^{I}\right)}{\left(\Delta A_{C} \sqrt{\mathcal{I}_{F}^{C}}+\Delta A_{I} \sqrt{\mathcal{I}_{F}^{I}}\right)^{2}}} \\
& =\sqrt{\frac{\left(\Delta A_{C}\right)^{2} \mathcal{I}_{F}^{C}+\left(\Delta A_{I}\right)^{2} \mathcal{I}_{F}^{I}+\left(\Delta A_{C}\right)^{2} \mathcal{I}_{F}^{I}+\left(\Delta A_{I}\right)^{2} \mathcal{I}_{F}^{C}}{\left(\Delta A_{C} \sqrt{\mathcal{I}_{F}^{C}}+\Delta A_{I} \sqrt{\mathcal{I}_{F}^{I}}\right)^{2}}} \\
& =\sqrt{\frac{\left(\Delta A_{C} \sqrt{\mathcal{I}_{F}^{C}}+\Delta A_{I} \sqrt{\mathcal{I}_{F}^{I}}\right)^{2}-2 \Delta A_{C} \Delta A_{I} \sqrt{\mathcal{I}_{F}^{C} \mathcal{I}_{F}^{I}}+\left(\Delta A_{C}\right)^{2} \mathcal{I}_{F}^{I}+\left(\Delta A_{I}\right)^{2} \mathcal{I}_{F}^{C}}{\left(\Delta A_{C} \sqrt{\mathcal{I}_{F}^{C}}+\Delta A_{I} \sqrt{\mathcal{I}_{F}^{I}}\right)^{2}}} \\
& =\sqrt{1+\frac{\left(\Delta A_{C} \sqrt{\mathcal{I}_{F}^{I}}-\Delta A_{I} \sqrt{\mathcal{I}_{F}^{C}}\right)^{2}}{\left(\Delta A_{C} \sqrt{\mathcal{I}_{F}^{C}}+\Delta A_{I} \sqrt{\mathcal{I}_{F}^{I}}\right)^{2}}} \geq 1
\end{aligned}
$$

proving Eq. (17) in the main text. This shows that the coherent-incoherent bound (F2) is tighter than Eq. (F1) [bound (12) is tighter than Eq. (3) in the main text]. The bounds coincide only when $\Delta A_{C} \sqrt{\mathcal{I}_{F}^{I}}=\Delta A_{I} \sqrt{\mathcal{I}_{F}^{C}}$.

\section{APPENDIX G: COMPARING THE BOUNDS ON A QUBIT}

In this appendix, we present detailed derivations for the last two paragraphs of Sec. V and for Fig. 1 in the main text. Specifically, we construct operators that saturate the 
coherent and incoherent bounds for a qubit suffering dephasing and use these results to compare bounds (3), (10), and (12) in the main text.

Let us consider a qubit with Hamiltonian

$$
H=\frac{\omega}{2} \sigma_{y}
$$

and state

$$
\rho_{t}=\frac{\mathbb{1}+x \sigma_{x}+z \sigma_{z}}{2}
$$

constrained to the $y=\operatorname{Tr}\left(\rho \sigma_{y}\right)=0$ plane, with $x=$ $\operatorname{Tr}\left(\rho \sigma_{x}\right)$ and $z=\operatorname{Tr}\left(\rho \sigma_{z}\right)$.

We have

$$
\begin{aligned}
L_{C} & =-2 i \sum_{j \neq k} \frac{\left\langle j\left|\left[H_{t}, \rho_{t}\right]\right| k\right\rangle}{\left(p_{j}+p_{k}\right)}|j\rangle\left\langle k\left|=\omega \sum_{j \neq k} \frac{\left\langle j\left|\left(-x \sigma_{z}+z \sigma_{x}\right)\right| k\right\rangle}{\left(p_{j}+p_{k}\right)}\right| j\right\rangle\left\langle k\left|=\omega \sum_{j \neq k}\left\langle j\left|\left(-x \sigma_{z}+z \sigma_{x}\right)\right| k\right\rangle\right| j\right\rangle\langle k| \\
& =\omega \sum_{j k}\left\langle j\left|\left(-x \sigma_{z}+z \sigma_{x}\right)\right| k\right\rangle|j\rangle\left\langle k\left|-\omega \sum_{j}\left\langle j\left|\left(-x \sigma_{z}+z \sigma_{x}\right)\right| j\right\rangle\right| j\right\rangle\langle j| \\
& =\omega\left(-x \sigma_{z}+z \sigma_{x}\right)-\omega \sum_{j}\left\langle j\left|\left(-x \sigma_{z}+z \sigma_{x}\right)\right| j\right\rangle|j\rangle\langle j|,
\end{aligned}
$$

where we used the fact that, for a two-level system, $p_{1}+p_{2}=1$.

Then, the operator $\sigma_{y}$ satisfies

$$
\begin{aligned}
\operatorname{cov}\left(L_{C}, \sigma_{y}\right) & =\frac{1}{2} \omega \operatorname{Tr}\left(\rho_{t}\left\{-x \sigma_{z}+z \sigma_{x}, \sigma_{y}\right\}\right) \\
& -\frac{1}{2} \omega \sum_{j}\left\langle j\left|\left(-x \sigma_{z}+z \sigma_{x}\right)\right| j\right\rangle \operatorname{Tr}\left(\rho_{t}\left\{|j\rangle\langle j|, \sigma_{y}\right\}\right) \\
& =0,
\end{aligned}
$$

where we used the fact that $\left\{\sigma_{x}, \sigma_{y}\right\}=\left\{\sigma_{z}, \sigma_{y}\right\}=0$ and that the eigenvectors $\{|j\rangle\}$ of $\rho_{t}$ belong to the $y=0$ plane, which implies that $\operatorname{Tr}\left(\rho_{t}\left\{|j\rangle\langle j|, \sigma_{y}\right\}\right)=0$. Note that this can also be concluded directly from the fact that, under unitary dynamics, $\dot{y}=\operatorname{cov}\left(L_{C}, \sigma_{y}\right)$ and that, for the chosen initial state and Hamiltonian, we have $\dot{y}=0$.

Now, consider a nonunitary term in the dynamics causing dephasing along $\sigma_{z}$ with a rate $\kappa$, modeled by $U_{t}\left(d \chi_{t} / d t\right) U_{t}^{\dagger}=-\kappa\left[\sigma_{z},\left[\sigma_{z}, \rho_{t}\right]\right]$ in Eq. (6) in the main text. This leaves the qubit to be constrained to the $y=0$ plane as well. The operator $\sigma_{y}$ then satisfies

$\operatorname{cov}\left(L_{I}, \sigma_{y}\right)=\sum_{j} p_{j} \frac{\dot{p}_{j}}{p_{j}}\left\langle j\left|\sigma_{y}\right| j\right\rangle=\sum_{j} \dot{p}_{j}\left\langle j\left|\sigma_{y}\right| j\right\rangle=0$

Given that $\operatorname{cov}\left(L_{C}, \mathbb{1}\right)=\operatorname{cov}\left(L_{I}, \mathbb{1}\right)=0$, this implies that the set $\left\{L_{C}, L_{I}, \sigma_{y}, \mathbb{1}\right\}$ forms a complete basis of orthogonal operators. The operators $\mathbb{1}$ and $\sigma_{y}$ are thus "still"; i.e., they evolve neither under the Hamiltonian nor under the dephasing. Moreover, since $\sigma_{x}$ and $\sigma_{z}$ are orthogonal to $\sigma_{y}$ and $\mathbb{1}$, the former two can be written solely in terms of the preferred "speed" operators as

$$
\sigma_{z}=\alpha_{C}^{z} L_{C}+\alpha_{I}^{z} L_{I}, \quad \sigma_{x}=\alpha_{C}^{x} L_{C}+\alpha_{I}^{x} L_{I} .
$$

Observables $\sigma_{z}$ and $\sigma_{x}$ thus saturate the coherent and incoherent bounds (10) in the main text. In the case of unitary dynamics $(\kappa=0)$, we have $L_{I}=0$ and $L=L_{C}$, which means that the upper bound (3) in the main text is also saturated. Finally, saturation of the upper bound (12) depends on the relative signs of $\alpha_{C}$ and $\alpha_{I}$ : When $\operatorname{sign}\left(\alpha_{C}\right) \neq \operatorname{sign}\left(\alpha_{I}\right)$, Eq. (12) is not saturated.

\section{APPENDIX H: BOUND IN TERMS OF PATH DIVERGENCES}

In this appendix, we derive bounds on integrated changes in an observable in terms of path divergences. We prove Eq. (20) of Sec. VI in the main text and show how to upper bound Eqs. (21) and (22) in terms of coherent and incoherent path divergences.

The divergence of a path is defined by $[69,92,93]$

$$
\mathcal{J}\left(\rho_{i}, \rho_{f}\right):=\tau \int_{0}^{\tau} \mathcal{I}_{F} d t
$$

and is related to the energy of a path, $\mathcal{J} / 2 \tau$. Note that the square of the length of a path is upper bounded by the path's divergence: 


$$
\begin{aligned}
\mathcal{L}\left(\rho_{i}, \rho_{f}\right) & =\int_{0}^{\tau} \sqrt{\mathcal{I}_{F}} d t=\tau \frac{1}{\tau} \int_{0}^{\tau} \sqrt{\mathcal{I}_{F}} d t \leq \tau \sqrt{\frac{1}{\tau} \int_{0}^{\tau} \mathcal{I}_{F} d t} \\
& =\tau \sqrt{\frac{1}{\tau^{2}} \mathcal{J}\left(\rho_{i}, \rho_{f}\right)}=\sqrt{\mathcal{J}\left(\rho_{i}, \rho_{f}\right)} .
\end{aligned}
$$

Equations (3) and (18), and the Cauchy-Schwarz inequality lead to an integrated bound

$$
\begin{aligned}
|a(\tau)| & =\left|\int_{0}^{\tau} \dot{a} d t\right| \leq 2 \int_{0}^{\tau} \sqrt{\mathcal{I}_{F}} \Delta A d t \\
& =2 \tau \frac{1}{\tau} \int_{0}^{\tau} \sqrt{\mathcal{I}_{F}} \Delta A d t \\
& \leq 2 \tau \sqrt{\frac{1}{\tau} \int_{0}^{\tau} \mathcal{I}_{F} d t} \sqrt{\frac{1}{\tau} \int_{0}^{\tau}(\Delta A)^{2} d t .}
\end{aligned}
$$

Then, the total change in an observable is bounded by the divergence of the path in Hilbert space and the integrated fluctuations in the observable:

$$
\begin{aligned}
|a(\tau)|^{2} & \leq 4 \tau^{2}\left(\frac{1}{\tau} \int_{0}^{\tau} \mathcal{I}_{F} d t\right)\left(\frac{1}{\tau} \int_{0}^{\tau}(\Delta A)^{2} d t^{\prime}\right) \\
& =4 \mathcal{J}\left(\rho_{i}, \rho_{f}\right) \frac{1}{\tau} \int_{0}^{\tau}(\Delta A)^{2} d t
\end{aligned}
$$

This proves Eq. (20) in the main text.

The divergence of a path can be decomposed into coherent and incoherent terms, $\mathcal{J}\left(\rho_{i}, \rho_{f}\right)=\mathcal{J}_{C}\left(\rho_{i}, \rho_{f}\right)+$ $\mathcal{J}_{I}\left(\rho_{i}, \rho_{f}\right)$, with

$\mathcal{J}_{C}\left(\rho_{i}, \rho_{f}\right):=\tau \int_{0}^{\tau} \mathcal{I}_{F}^{C} d t \quad$ and $\quad \mathcal{J}_{I}\left(\rho_{i}, \rho_{f}\right):=\tau \int_{0}^{\tau} \mathcal{I}_{F}^{I} d t$.

The total changes in the observable due to coherent and incoherent dynamics are bounded by

$$
\begin{aligned}
& \left|a(\tau)-a_{I}(\tau)\right| \leq 2 \sqrt{\mathcal{J}_{C}\left(\rho_{i}, \rho_{f}\right) \frac{1}{\tau} \int_{0}^{\tau}\left(\Delta A_{C}\right)^{2}} d t, \\
& \left|a(\tau)-a_{C}(\tau)\right| \leq 2 \sqrt{\mathcal{J}_{I}\left(\rho_{i}, \rho_{f}\right) \frac{1}{\tau} \int_{0}^{\tau}\left(\Delta A_{I}\right)^{2}} d t .
\end{aligned}
$$

This shows how to upper bound Eqs. (21) and (22) in the main text in terms of coherent and incoherent path divergences.

\section{APPENDIX I: COHERENT SPEEDUP OF INCOHERENT PROCESSES}

In this appendix, we derive the Hamiltonian in Eq. (23) of Sec. VI in the main text, which drives an observable at the maximum allowed speed. We then use this Hamiltonian to enhance an incoherent process on a qubit, presenting detailed derivations for the two paragraphs following Eq. (23).

What Hamiltonian induces the fastest change to an observable $A$ ? The speed with which the observable changes due to coherent drive satisfies $\dot{a}_{C}=\operatorname{cov}\left(L_{C}, A_{C}\right) \leq$ $\Delta L_{C} \Delta A_{C}$. Thus, any Hamiltonian that leads to a symmetric logarithmic derivative for which $L_{C} \propto A_{C}$ will saturate the speed limit and drive the observable as fast as allowed by nature. Using Eq. (8a), a direct calculation shows that the Hamiltonian [Eq. (23) in the main text]

$$
H_{t}^{\text {speedup }}:=-\lambda_{t} \frac{i}{2} \sum_{j \neq k} \frac{\left(p_{j}+p_{k}\right)}{p_{j}-p_{k}} A_{j k}|j\rangle\langle k|
$$

leads to

$$
L_{C}^{\text {speedup }}=\lambda_{t} A_{C} .
$$

This Hamiltonian thus induces dynamics for which the coherent bound for observable $A$ saturates. The prefactor $\lambda_{t}$ sets the energy scale of the Hamiltonian and would typically be constrained by the available resources. Note that the Hamiltonian $H_{t}^{\text {speedup }}$ that leads the observable to evolve at the speed limit is state and observable dependent-it is a Hamiltonian tailored to drive the expectation value of the observable as rapidly as possible given the available resources.

The observable evolves according to

$\dot{a}_{C}=\operatorname{cov}\left(A_{C}, L_{C}\right)=\Delta A_{C} \Delta L_{C}^{\text {speedup }}=\lambda_{t}\left(\Delta A_{C}\right)^{2}=\frac{\mathcal{I}_{F}^{C}}{\lambda_{t}}$,

and the integrated change in the observable due to coherent dynamics becomes

$$
\begin{aligned}
\left|a(\tau)-a_{I}(\tau)\right| & =\left|a_{C}(\tau)\right|=\left|\int_{0}^{\tau} \operatorname{cov}\left(A_{C}, L_{C}^{\text {speedup }}\right) d t\right| \\
& =\int_{0}^{\tau} \lambda_{t}\left(\Delta A_{C}\right)^{2} d t=\int_{0}^{\tau} \frac{\mathcal{I}_{F}^{C}}{\lambda_{t}} d t .
\end{aligned}
$$

This coherent drive can be used to enhance incoherent processes, up to an amount characterized by the integrated coherent Fisher information weighed by the scaling prefactor $\lambda_{t}$.

We consider a toy model of an incoherent erasure process of a qubit [110]. In an auxiliary reset state $|r\rangle$, the expectation value of all qubit observables is null, e.g., $\left\langle\sigma_{x}\right\rangle=\left\langle\sigma_{y}\right\rangle=\left\langle\sigma_{z}\right\rangle=0$. Incoherent dynamics drives the system to such an erased state with a rate $\gamma$. We model this incoherent dynamics by a Lindblad master equation with jump operators $L_{0}:=|r\rangle\langle 0|$ and $L_{1}:=|r\rangle\langle 1|$, 


$$
\begin{aligned}
\dot{\rho}_{t} & =\gamma \sum_{j=0,1}\left(L_{j} \rho_{t} L_{j}^{\dagger}-\frac{1}{2}\left\{L_{j}^{\dagger} L_{j}, \rho_{t}\right\}\right) \\
& =\gamma\left(\operatorname{Tr}\left(\rho_{t} \Pi_{2}\right)|r\rangle\langle r|-\frac{1}{2}\left\{\Pi_{2}, \rho_{t}\right\}\right),
\end{aligned}
$$

where we define the projector $\Pi_{2}:=|0\rangle\langle 0|+| 1\rangle\langle 1|$ onto the subspace of the two-level system.

For illustration purposes, we suppose that it is more critical to erase certain aspects of the information stored in the initial state $\left|\Psi_{0}\right\rangle=a|0\rangle+b|1\rangle$ of the two-level system. For instance, this could be because it is expected that an adversary will attempt to acquire information about the initial value $z_{0}:=\left\langle\sigma_{z}\right\rangle(0)=a-b$, by measuring $\sigma_{z}$ on an ensemble of such effective qubits, each one initially prepared in $\left|\Psi_{0}\right\rangle$. We wish to hide the fact that $z_{0} \neq 0$.

Under the incoherent erasure process, information of $z_{0}$ is exponentially erased at a rate $\gamma$,

$$
\begin{aligned}
\dot{z}_{t}^{\text {incoh }} & =\operatorname{Tr}\left(\sigma_{z} \dot{\rho}_{t}\right) \\
& =\gamma\left(\operatorname{Tr}\left(\rho_{t} \Pi_{2}\right)\left\langle r\left|\sigma_{z}\right| r\right\rangle-\frac{1}{2} \operatorname{Tr}\left(\left\{\sigma_{z}, \Pi_{2}\right\} \rho_{t}\right)\right) \\
& =-\gamma \operatorname{Tr}\left(\sigma_{z} \rho_{t}\right)=-\gamma z_{t}^{\text {incoh }},
\end{aligned}
$$

where we used $\left\langle r\left|\sigma_{z}\right| r\right\rangle=0$.

We can take advantage of a coherent drive to enhance the process of hiding the fact that $z_{0} \neq 0$ from the adversary. Hamiltonian $H_{t}^{\text {speedup }}$ in Eq. (I1) [Eq. (23) in the main text] defines the fastest time-local way to do this. Given that $\sigma_{z}|r\rangle=0$, at time $t=0$ the Hamiltonian $H_{t}^{\text {speedup }}$ in Eq. (I1) does not connect the reset state $|r\rangle$ to states $|0\rangle$ and $|1\rangle$. This implies that $|r\rangle$ remains an eigenstate of $\rho_{t}$ under the action of the incoherent dynamics as well as the coherent dynamics. Moreover, since dynamics is coherent within the qubit subspace, we can denote the remaining eigenstates of the evolved state by $\left|\Psi_{t}\right\rangle$ and $\left|\Psi_{t}^{\perp}\right\rangle$ with eigenvalues $p_{\Psi}$ and 0 . The state then takes the form $\rho_{t}=p_{\Psi}(t)\left|\Psi_{t}\right\rangle\left\langle\Psi_{t}\left|+\left(1-p_{\Psi}(t)\right)\right| r\right\rangle\langle r|$. Therefore, the speedup Hamiltonian becomes

$$
\begin{aligned}
H_{t}^{\text {speedup }} & :=\operatorname{sign}\left(z_{t}\right) \lambda_{t} \frac{i}{2} \sum_{j \neq k} \frac{\left(p_{j}+p_{k}\right)}{p_{j}-p_{k}} A_{j k}|j\rangle\langle k| \\
& =\operatorname{sign}\left(z_{t}\right) \lambda_{t} \frac{i}{2}\left(\frac{p_{\Psi}+0}{p_{\Psi}-0}\left\langle\Psi_{t}\left|\sigma_{z}\right| \Psi_{t}^{\perp}\right\rangle\left|\Psi_{t}\right\rangle\left\langle\Psi_{t}^{\perp}\left|+\frac{0+p_{\Psi}}{0-p_{\Psi}}\left\langle\Psi_{t}^{\perp}\left|\sigma_{z}\right| \Psi_{t}\right\rangle\right| \Psi_{t}^{\perp}\right\rangle\left\langle\Psi_{t}\right|\right) \\
& =\operatorname{sign}\left(z_{t}\right) \lambda_{t} \frac{i}{2}\left(\left\langle\Psi_{t}\left|\sigma_{z}\right| \Psi_{t}^{\perp}\right\rangle\left|\Psi_{t}\right\rangle\left\langle\Psi_{t}^{\perp}\left|-\left\langle\Psi_{t}^{\perp}\left|\sigma_{z}\right| \Psi_{t}\right\rangle\right| \Psi_{t}^{\perp}\right\rangle\left\langle\Psi_{t}\right|\right),
\end{aligned}
$$

where $A=\sigma_{z}$, and we have chosen the sign relative to Eq. (I1) to ensure that the coherent dynamics drives the system with $\dot{z}_{t} / z_{t} \leq 0$, i.e., helps hide the fact that $z_{0} \neq 0$ by driving $\left\langle\sigma_{z}\right\rangle$ towards 0 . Indeed, with the chosen sign in Eq. (I7), we have $L_{C}^{\text {speedup }}=-\operatorname{sign}\left(z_{t}\right) \lambda_{t} \sigma_{(z, C)}$, and the incoherent drive enforces

$$
\dot{z}_{C}=\operatorname{cov}\left(\sigma_{(z, C)}, L_{C}^{\text {speedup }}\right)=-\operatorname{sign}\left(z_{t}\right) \frac{\mathcal{I}_{F}^{C}}{\lambda_{t}} .
$$

$$
\left\langle\Psi_{t}^{\perp}\left|\sigma_{z}\right| \Psi_{t}\right\rangle=\left\langle\Psi_{t}\left|\sigma_{z}\right| \Psi_{t}^{\perp}\right\rangle=-2 a_{t} b_{t}=-x_{t} .
$$

Then,

$$
\begin{aligned}
H_{t}^{\text {speedup }} & =\operatorname{sign}\left(z_{t}\right) \lambda_{t} \frac{i}{2}\left(-x_{t}\left|\Psi_{t}\right\rangle\left\langle\Psi_{t}^{\perp}\left|+x_{t}\right| \Psi_{t}^{\perp}\right\rangle\left\langle\Psi_{t}\right|\right)=-\operatorname{sign}\left(z_{t}\right) \lambda_{t} \frac{i}{2} x_{t}\left(\left|\Psi_{t}\right\rangle\left\langle\Psi_{t}^{\perp}|-| \Psi_{t}^{\perp}\right\rangle\left\langle\Psi_{t}\right|\right) \\
& =-\operatorname{sign}\left(z_{t}\right) \lambda_{t} \frac{i}{2} x_{t}\left(-\left(a_{t}^{2}+b_{t}^{2}\right)|0\rangle\left\langle 1\left|+\left(a_{t}^{2}+b_{t}^{2}\right)\right| 1\right\rangle\langle 0|\right)=-\operatorname{sign}\left(z_{t}\right) \lambda_{t} \frac{x_{t}}{2}(-i|0\rangle\langle 1|+i| 1\rangle\langle 0|) \\
& =\operatorname{sign}\left(z_{t}\right) \lambda_{t} \frac{x_{t}}{2} \sigma_{y} .
\end{aligned}
$$


The coefficient $\lambda_{t}$ is to be defined by the resources available to drive our coherent dynamics. If, for instance, we set the spectral norm of the Hamiltonian to satisfy $\left\|H_{t}^{\text {speedup }}\right\|=\epsilon$, we obtain $\lambda_{t}=\left(2 \epsilon /\left|x_{t}\right|\right)$. Then, we find that the normalized optimal Hamiltonian becomes

$$
H_{t}^{\text {speedup }}=\epsilon \operatorname{sign}\left(z_{t}\right) \frac{x_{t}}{\left|x_{t}\right|} \sigma_{y}=\epsilon \frac{\operatorname{sign}\left(z_{t}\right)}{\operatorname{sign}\left(x_{t}\right)} \sigma_{y} .
$$

At any time, this Hamiltonian enhances the hiding process as much as allowed by coherent dynamics.

With this result, we can compare the rates of erasure of the incoherent and the coherently enhanced dynamics. At any time $t$, the coherently enhanced state evolves following

$$
\dot{\rho}_{t}=-i\left[H_{t}^{\text {speedup }}, \rho_{t}\right]+\gamma\left(\operatorname{Tr}\left(\rho_{t} \Pi_{2}\right)|r\rangle\langle r|-\frac{1}{2}\left\{\Pi_{2}, \rho_{t}\right\}\right) .
$$

Thus, the rate of change in $z_{t}$ satisfies

$$
\begin{aligned}
\dot{z}_{t} & =-i \operatorname{Tr}\left(\left[\sigma_{z}, H_{t}^{\text {speedup }}\right] \rho_{t}\right)-\gamma z_{t} \\
& =-i \epsilon \frac{\operatorname{sign}\left(z_{t}\right)}{\operatorname{sign}\left(x_{t}\right)} \operatorname{Tr}\left(\left[\sigma_{z}, \sigma_{y}\right] \rho_{t}\right)-\gamma z_{t} \\
& =-2 \epsilon \frac{\operatorname{sign}\left(z_{t}\right)}{\operatorname{sign}\left(x_{t}\right)}\left\langle\sigma_{x}\right\rangle-\gamma z_{t},
\end{aligned}
$$

which means that

$$
\dot{z}_{t} / z_{t}=-\gamma-2 \epsilon\left|\frac{x_{t}}{z_{t}}\right| .
$$

This process is faster than the one from the purely incoherent erasure process in Eq. (I6), $\dot{z}_{t}^{\text {incoh }}=-\gamma z_{t}^{\text {incoh }}$. This proves the claim in the two paragraphs following Eq. (23) of Sec. VI in the main text.

\section{APPENDIX J: LIMITS TO SPEED IN HILBERT SPACE}

In this appendix, we derive speed limits in state space from the coherent-incoherent bounds on observables, placing upper bounds on the rate of change of the quantum fidelity $F\left(\rho_{t}, \rho_{0}\right)$ between the initial and evolved states, referred to after Eq. (18) in the main text and Ref. [94].

We assume a pure initial state, $\rho_{0}^{2}=\rho_{0}=\chi_{0}$. In this case, the fidelity between the initial and evolved states becomes $F_{t}:=F\left(\rho_{t}, \rho_{0}\right):=\left(\operatorname{Tr}\left(\sqrt{\sqrt{\rho_{t}} \rho_{0} \sqrt{\rho_{t}}}\right)\right)^{2}=\operatorname{Tr}\left(\rho_{0} \rho_{t}\right)$. Taking $A=\rho_{0}$ in Eqs. (10) and (12), $F_{t}$ satisfies

$$
\begin{aligned}
\left|\frac{d}{d t} F_{t}\right| & =\left|\operatorname{Tr}\left(\rho_{0} \frac{d}{d t} \rho_{t}\right)\right| \\
& =\left|\operatorname{cov}\left(\rho_{C}, L_{C}\right)+\operatorname{cov}\left(\rho_{I}, L_{I}\right)\right| \\
& \leq \Delta \rho_{C} \sqrt{\mathcal{I}_{F}^{C}}+\Delta \rho_{I} \sqrt{\mathcal{I}_{F}^{I}},
\end{aligned}
$$

where

$$
\rho_{C}=\sum_{j \neq k} \rho_{0, j k}|j\rangle\left\langle k\left|, \quad \rho_{I}=\sum_{j} \rho_{0, j}\right| j\right\rangle\langle j|
$$

are the coherent and incoherent components of the initial state $\rho_{0}$ in the eigenbasis of the evolved state $\rho_{t}$.

From Appendix F, we also have

$$
\left|\frac{d}{d t} F_{t}\right| \leq \Delta \rho_{C} \sqrt{\mathcal{I}_{F}^{C}}+\Delta \rho_{I} \sqrt{\mathcal{I}_{F}^{I}} \leq \Delta \rho_{0} \sqrt{\mathcal{I}_{F}},
$$

with a weaker bound in terms of the Fisher information $\mathcal{I}_{F}$. Using

$$
\begin{gathered}
\left(\Delta \rho_{C}\right)^{2}=\left(\Delta \rho_{0}\right)^{2}-\left(\Delta \rho_{I}\right)^{2}, \\
\left(\Delta \rho_{0}\right)^{2}=\operatorname{Tr}\left(\rho_{t} \rho_{0}^{2}\right)-\left(\operatorname{Tr}\left(\rho_{t} \rho_{0}\right)\right)^{2}=F_{t}-F_{t}^{2},
\end{gathered}
$$

we obtain

$\frac{\left|\frac{d}{d t} F_{t}\right|}{\sqrt{F_{t}-F_{t}^{2}}} \leq \sqrt{1-\frac{\left(\Delta \rho_{I}\right)^{2}}{F_{t}-F_{t}^{2}}} \sqrt{\mathcal{I}_{F}^{C}}+\sqrt{\frac{\left(\Delta \rho_{I}\right)^{2}}{F_{t}-F_{t}^{2}}} \sqrt{\mathcal{I}_{F}^{C}} \leq \sqrt{\mathcal{I}_{F}}$.

Upon integration, this gives a bound on the total change in fidelity:

$$
\begin{aligned}
\arccos \left(\sqrt{F\left(\rho_{\tau}, \rho_{0}\right)}\right) \leq & \int_{0}^{\tau} \sqrt{1-\frac{\left(\Delta \rho_{I}\right)^{2}}{F_{t}-F_{t}^{2}}} \sqrt{\frac{\mathcal{I}_{F}^{C}}{4}} \\
& +\sqrt{\frac{\left(\Delta \rho_{I}\right)^{2}}{F_{t}-F_{t}^{2}}} \sqrt{\frac{\mathcal{I}_{F}^{C}}{4}} d t \\
& \leq \int_{0}^{\tau} \sqrt{\frac{\mathcal{I}_{F}}{4}} d t .
\end{aligned}
$$

The rightmost bound is the one derived in Ref. [18]. The tighter intermediate bound is made possible by singling out the coherent and incoherent effects on the change in the fidelity. The two bounds coincide only for purely coherent dynamics of a quantum system.

\section{APPENDIX K: COMPARISON TO BOUNDS FROM PARAMETER ESTIMATION THEORY}

In this appendix, we compare Eq. (12) in Sec. III of the main text to the speed limits that can be derived from the 
quantum Cramér-Rao bound. We also show how to recover Eq. (3) in Sec. II from the quantum Cramér-Rao bound.

\section{Quantum Cramér-Rao bound}

The quantum Cramér-Rao bound generalizes the Cramér-Rao bound from classical estimation theory to quantum systems [51-53,111]. When estimating a parameter $\lambda$ on a system in state $\rho(\lambda)$, the standard deviation of any estimator $\hat{\lambda}$ of the parameter $\lambda$ satisfies [53]

$$
\frac{\Delta \hat{\lambda}}{\left|\frac{d}{d \lambda}\langle\hat{\lambda}\rangle\right|} \geq \frac{1}{\sqrt{\mathcal{I}_{F}}} .
$$

The quantum Fisher information is

$$
\mathcal{I}_{F}:=2 \sum_{j k} \frac{\left|\left\langle j\left|\frac{\partial \rho(\lambda)}{\partial \lambda}\right| k\right\rangle\right|^{2}}{p_{j}+p_{k}},
$$

with a summation over indices such that $p_{j}+p_{k} \neq 0$. The bound is achievable for the optimal estimator [53]. Note that the bound assumes that the estimator $\hat{\lambda}$ is independent of the parameter $\lambda$ to be estimated $[53,58,60]$ (we discuss this further in Appendix K 2 below).

The quantum parameter estimation problem involves two optimizations: (i) optimizing over all possible observables that can be measured-more generally, optimizing over all possible positive operator valued measures (POVMs) that can be performed-and (ii) optimizing over all possible estimators $\hat{\lambda}$ that can be constructed from the measurement outcomes. Optimization (ii) is accounted for by the classical Cramér-Rao bound, but (i) entails a purely quantum aspect to the problem.

\section{Restricting the estimator to functions of $\langle A\rangle$}

In this paper, we focus on speed limits, i.e., on the rate of change of the expectation value of an observable $A$. The Cramér-Rao bound can also be cast as a bound on this rate of change.

Focusing on the case of time as the parameter $\lambda=t$ to be estimated, Eq. (K1) imposes a bound on the rate of change of the mean of any estimator $\hat{t}$ of $t$. If one restricts to a timeindependent observable $\hat{t}=A$, the Cramér-Rao bound implies

$$
\left|\frac{d}{d t}\langle A\rangle\right| \leq \sqrt{\mathcal{I}_{F}} \Delta A .
$$

For the case of operators without explicit time dependence, bound (3) in the main text coincides with bound (K3) implied by the quantum Cramér-Rao theorem. The CramérRao bound assumes no time parameter dependence in the estimators [53] though, so it does not directly recover (3) for time-dependent operators. However, the following procedure allows time-dependent operators. We wish to find a speed limit at time $t=t_{0}$ for the expectation value $\operatorname{Tr}\left(\rho_{t} A(t)\right)$ of an operator $A(t)$ (to avoid confusion, we write the explicit time dependence for the proof that follows). Bound (K3) applies to any operator-in particular, to $A\left(t_{0}\right)$ - and implies

$$
\left|\frac{d}{d t} \operatorname{Tr}\left(\rho_{t} A\left(t_{0}\right)\right)\right| \leq \sqrt{\mathcal{I}_{F}(t)} \Delta A\left(t_{0}\right) .
$$

Evaluating this bound at $t=t_{0}$ recovers bound (3) in the main text.

\section{Identifying coherent and incoherent contributions to the dynamics}

The restriction to a specific observable and the specification of the evolution of the state that singles out contributions from unitary and incoherent dynamics enable the main bounds in this article: upper bounds (10) and (12). As we prove in Appendix F, the latter bound is tighter than the speed limit (K3) derivable from the quantum CramérRao bound.

Moreover, we stress that the lower speed bounds (11) are not accounted for by Cramér-Rao bounds but are instead made possible by the separation of the dynamics into two terms, which in turn allows for applying the reverse triangle inequality.

\section{An alternative bound with basis-dependent classical Fisher information}

A possible classical parameter estimation problem is to forego optimization (i) in the quantum Cramér-Rao bound above and instead restrict to a specific measurement basis [see discussion after Eq. (K1)]. Then, for measurements performed in a fixed basis $\left\{\Pi_{\alpha}=\left|\phi_{\alpha}\right\rangle\left\langle\phi_{\alpha}\right|\right\}$ with outcome probabilities $q_{\alpha}^{\Pi}=\operatorname{Tr}\left(\rho \Pi_{\alpha}\right)$, this recovers a classical parameter estimation problem. One can obtain classical Fisher information from the probabilities $\left\{q_{\alpha}^{\Pi}\right\}$ :

$$
F_{\Pi}:=\sum_{\alpha} q_{\alpha}^{\Pi}\left(\frac{d}{d t} \ln q_{\alpha}^{\Pi}\right)^{2} .
$$

The classical Cramér-Rao bound thus says that, for any estimator $\hat{t}$ constructed from outcomes of the POVM $\left\{\Pi_{\alpha}\right\}$, it holds that [112-114]

$$
\left|\frac{d}{d t}\langle\hat{t}\rangle\right| \leq \sqrt{F_{\Pi}} \Delta \hat{t}
$$

If measurements are performed solely in the restricted measurement basis, this bound is tighter than the quantum Cramér-Rao bound (K1) since the latter is valid for any measurement basis. Bound (K5) involves optimization (ii) over all possible estimators, given the restricted measurement basis $\left\{\Pi_{\alpha}\right\}$. 
Further restricting to $\hat{t}=A=\sum_{\alpha} a_{\alpha} \Pi_{\alpha}$ gives

$$
\left|\frac{d}{d t}\langle A\rangle\right| \leq \sqrt{F_{\Pi}} \Delta A,
$$

which is in fact tighter than (K3) since $F_{\Pi} \leq \mathcal{I}_{F}$. In general, this bound will still be loose given that one is not performing optimization (ii) over all possible estimators.

The speed limit (K7) is distinct from our main new upper bound, Eq. (12) in the main text: There are cases in which Eq. (K7) is tighter than Eq. (12) and vice versa. In particular, if the measurement basis $\left\{\Pi_{\alpha}\right\}$ coincides with the optimal basis $\left\{\Pi_{\alpha}^{\text {optimal }}\right\}$ that saturates the quantum Cramér-Rao bound, then $F_{\Pi}=\mathcal{I}_{F}$ and Eq. (K7) coincides with Eq. (K3). In this case, Eq. (12) is tighter than Eq. (K7) (Appendix F). In contrast, there are also cases when Eq. (K7) is saturated while Eq. (12) is loose. For example, consider unitary dynamics under a Hamiltonian such that $\left[H, \Pi_{\alpha}\right]=0$, in which case $d\langle A\rangle / d t=0$ and $d q_{\alpha}^{\Pi} / d t=$ $\operatorname{Tr}\left(d \rho / d t \Pi_{\alpha}\right)=-i \operatorname{Tr}\left(\rho\left[\Pi_{\alpha}, H\right]\right)=0$. From Eq. (K5), we have that $F_{\Pi}=0$, and Eq. (K7) is trivially saturated while Eq. (12) is loose.

We emphasize, however, that the classical Fisher information (K5) explicitly depends on the measurement basis. In general, then, calculating $F_{\Pi}$ is more intricate and dependent on the system dynamics, the state, and a reference measurement basis. In contrast, $\mathcal{I}_{F}$ (as well as $\mathcal{I}_{F}^{C}$ and $\mathcal{I}_{F}^{I}$ ) are functions only of the state of the system and the dynamics that govern it.

When the measurement basis is chosen as the eigenbasis of the state of the system, $\left\{\Pi_{\alpha}\right\} \equiv\{|j\rangle\langle j|\}$, one obtains $\left\{q_{\alpha}^{\Pi}\right\} \equiv\left\{p_{j}\right\}$, so the classical basis-dependent Fisher information $F_{\Pi}$ coincides with $\mathcal{I}_{F}^{I}$ :

$$
F_{\Pi}=\sum_{j} p_{j}\left(\frac{d}{d t} \ln p_{j}\right)^{2}=\mathcal{I}_{F}^{I} .
$$

Then, if the estimator for $t$ is taken to be $\hat{t}=A_{I}=$ $\sum_{j} A_{j j}|j\rangle\langle j|$, the classical Cramér-Rao bound (K7) recovers the bound on the incoherent term in Eq. (10) of the main text:

$$
\left|\frac{d}{d t}\left\langle A_{I}\right\rangle\right| \leq \sqrt{\mathcal{I}_{F}^{I}} \Delta A_{I}
$$

for time-independent operators (which can be extended to time-dependent operators as was shown in Appendix K 2).

However, a similar trick to restrict the basis does not work on the bound for the coherent term $A_{C}$ since the eigenbasis of $A_{C}$ does not commute with that of $\rho$. A classical parameter estimation bound on $A_{C}$ would then yield basis-dependent classical Fisher information.

This discussion results in a set of bounds that depend on the level of optimization involved in them. The main upper bounds in this article-Eqs. (10) and (12)—are tailored to the problem of the speed of evolution of an observable, and they give a provably tighter upper speed limit than the one implied by the quantum Cramér-Rao bound. In contrast, the quantum Cramér-Rao bound focuses on a different question that, in the context of parameter estimation theory, involves an optimization over all possible estimators of a parameter. As such, the quantum Cramér-Rao bound implies a looser speed limit on $\langle A\rangle$ as a result. Adding further ingredients to the problem, such as information of the outcome probabilities in the eigenbasis of the observable of interest [Eq. (K5)], can result in distinct (potentially tighter) bounds. The trade-off is that more information may be needed to evaluate the bounds, as in the case of Eq. (K7).

[1] L. Mandelstam and I. Tamm, The Uncertainty Relation between Energy and Time in Nonrelativistic Quantum Mechanics, J. Phys. (USSR) 9, 1 (1945).

[2] N. Margolus and L. B. Levitin, The Maximum Speed of Dynamical Evolution, Physica D (Amsterdam) 120, 188 (1998).

[3] B. Zieliński and M. Zych, Generalization of the MargolusLevitin Bound, Phys. Rev. A 74, 034301 (2006).

[4] F. Fröwis, Kind of Entanglement that Speeds Up Quantum Evolution, Phys. Rev. A 85, 052127 (2012).

[5] G. Tóth and I. Apellaniz, Quantum Metrology from a Quantum Information Science Perspective, J. Phys. A 47, 424006 (2014).

[6] A. Uhlmann, An Energy Dispersion Estimate, Phys. Lett. A 161, 329 (1992).

[7] A. K. Pati, New Derivation of the Geometric Phase, Phys. Lett. A 202, 40 (1995).

[8] V. Giovannetti, S. Lloyd, and L. Maccone, Quantum Limits to Dynamical Evolution, Phys. Rev. A 67, 052109 (2003).

[9] Y.-J. Zhang, W. Han, Y.-J. Xia, J.-P. Cao, and H. Fan, Quantum Speed Limit for Arbitrary Initial States, Sci. Rep. 4, 4890 (2014).

[10] D. P. Pires, M. Cianciaruso, L. C. Céleri, G. Adesso, and D. O. Soares-Pinto, Generalized Geometric Quantum Speed Limits, Phys. Rev. X 6, 021031 (2016).

[11] I. Marvian, R. W. Spekkens, and P. Zanardi, Quantum Speed Limits, Coherence, and Asymmetry, Phys. Rev. A 93, 052331 (2016).

[12] S. Deffner, Geometric Quantum Speed Limits: A Case for Wigner Phase Space, New J. Phys. 19, 103018 (2017).

[13] F. Campaioli, F. A. Pollock, F. C. Binder, and K. Modi, Tightening Quantum Speed Limits for Almost All States, Phys. Rev. Lett. 120, 060409 (2018).

[14] F. Campaioli, F. A. Pollock, and K. Modi, Tight, Robust, and Feasible Quantum Speed Limits for Open Dynamics, Quantum 3, 168 (2019).

[15] S. Sun and Y. Zheng, Distinct Bound of the Quantum Speed Limit via the Gauge Invariant Distance, Phys. Rev. Lett. 123, 180403 (2019).

[16] Z. Sun, J. Liu, J. Ma, and X. Wang, Quantum Speed Limits in Open Systems: Non-Markovian Dynamics without Rotating-Wave Approximation, Sci. Rep. 5, 8444 (2015). 
[17] Y. Shao, B. Liu, M. Zhang, H. Yuan, and J. Liu, Operational Definition of a Quantum Speed Limit, Phys. Rev. Research 2, 023299 (2020).

[18] M. M. Taddei, B. M. Escher, L. Davidovich, and R. L. de Matos Filho, Quantum Speed Limit for Physical Processes, Phys. Rev. Lett. 110, 050402 (2013).

[19] A. del Campo, I. L. Egusquiza, M. B. Plenio, and S. F. Huelga, Quantum Speed Limits in Open System Dynamics, Phys. Rev. Lett. 110, 050403 (2013).

[20] S. Deffner and E. Lutz, Quantum Speed Limit for Non-Markovian Dynamics, Phys. Rev. Lett. 111, 010402 (2013).

[21] L. P. García-Pintos and A. del Campo, Quantum Speed Limits under Continuous Quantum Measurements, New J. Phys. 21, 033012 (2019).

[22] T. Caneva, M. Murphy, T. Calarco, R. Fazio, S. Montangero, V. Giovannetti, and G. E. Santoro, Optimal Control at the Quantum Speed Limit, Phys. Rev. Lett. 103, 240501 (2009).

[23] S. Campbell and S. Deffner, Trade-off between Speed and Cost in Shortcuts to Adiabaticity, Phys. Rev. Lett. 118, 100601 (2017).

[24] K. Funo, J.-N. Zhang, C. Chatou, K. Kim, M. Ueda, and A. del Campo, Universal Work Fluctuations During Shortcuts to Adiabaticity by Counterdiabatic Driving, Phys. Rev. Lett. 118, 100602 (2017).

[25] S. Deffner, Energetic Cost of Hamiltonian Quantum Gates, Europhys. Lett. 134, 40002 (2021).

[26] S. Lloyd, Ultimate Physical Limits to Computation, Nature (London) 406, 1047 (2000).

[27] S. Lloyd, Computational Capacity of the Universe, Phys. Rev. Lett. 88, 237901 (2002).

[28] S. L. Braunstein, C. M. Caves, and G. J. Milburn, Generalized Uncertainty Relations: Theory, Examples, and Lorentz Invariance, Ann. Phys. (N.Y.) 247, 135 (1996).

[29] V. Giovannetti, S. Lloyd, and L. Maccone, Advances in Quantum Metrology, Nat. Photonics 5, 222 (2011).

[30] M. Beau and A. del Campo, Nonlinear Quantum Metrology of Many-Body Open Systems, Phys. Rev. Lett. 119, 010403 (2017).

[31] A. del Campo, J. Goold, and M. Paternostro, More Bang for Your Buck: Super-Adiabatic Quantum Engines, Sci. Rep. 4, 6208 (2014).

[32] F. Campaioli, F. A. Pollock, F. C. Binder, L. Céleri, J. Goold, S. Vinjanampathy, and K. Modi, Enhancing the Charging Power of Quantum Batteries, Phys. Rev. Lett. 118, 150601 (2017).

[33] K. Suzuki and K. Takahashi, Performance Evaluation of Adiabatic Quantum Computation via Quantum Speed Limits and Possible Applications to Many-Body Systems, Phys. Rev. Research 2, 032016(R) (2020).

[34] J. Jing, L.-A. Wu, and A. del Campo, Fundamental Speed Limits to the Generation of Quantumness, Sci. Rep. 6, 1 (2016).

[35] S. Deffner, Quantum Speed Limits and the Maximal Rate of Information Production, Phys. Rev. Research 2, 013161 (2020).

[36] D. P. Pires, K. Modi, and L. C. Céleri, Bounding Generalized Relative Entropies: Nonasymptotic Quantum Speed Limits, Phys. Rev. E 103, 032105 (2021).
[37] F. Campaioli, C. shui Yu, F. A. Pollock, and K. Modi, Resource Speed Limits: Maximal Rate of Resource Variation, arXiv:2004.03078.

[38] T. Fogarty, S. Deffner, T. Busch, and S. Campbell, Orthogonality Catastrophe as a Consequence of the Quantum Speed Limit, Phys. Rev. Lett. 124, 110601 (2020).

[39] A. del Campo, Probing Quantum Speed Limits with Ultracold Gases, Phys. Rev. Lett. 126, 180603 (2021).

[40] I. Marvian and D. A. Lidar, Quantum Speed Limits for Leakage and Decoherence, Phys. Rev. Lett. 115, 210402 (2015).

[41] N. Margolus, The Finite-State Character of Physical Dynamics, arXiv:1109.4994.

[42] B. Shanahan, A. Chenu, N. Margolus, and A. del Campo, Quantum Speed Limits across the Quantum-to-Classical Transition, Phys. Rev. Lett. 120, 070401 (2018).

[43] M. Okuyama and M. Ohzeki, Quantum Speed Limit Is Not Quantum, Phys. Rev. Lett. 120, 070402 (2018).

[44] V. T. Vo, T. Van Vu, and Y. Hasegawa, Unified Approach to Classical Speed Limit and Thermodynamic Uncertainty Relation, Phys. Rev. E 102, 062132 (2020).

[45] K. Ito and T. Miyadera, Fundamental Bound on the Power of Quantum Machines, arXiv:1711.02322.

[46] S. Julià-Farré, T. Salamon, A. Riera, M. N. Bera, and M. Lewenstein, Bounds on the Capacity and Power of Quantum Batteries, Phys. Rev. Research 2, 023113 (2020).

[47] L. P. García-Pintos, A. Hamma, and A. del Campo, Fluctuations in Extractable Work Bound the Charging Power of Quantum Batteries, Phys. Rev. Lett. 125, 040601 (2020).

[48] S. Deffner and S. Campbell, Quantum Speed Limits: From Heisenberg's Uncertainty Principle to Optimal Quantum Control, J. Phys. A 50, 453001 (2017).

[49] An interesting example where speed limits in Hilbert space correctly capture the dynamics of a many-body system is studied in Ref. [38].

[50] S. B. Nicholson, L. P. García-Pintos, A. del Campo, and J. R. Green, Time-Information Uncertainty Relations in Thermodynamics, Nat. Phys. 16, 1211 (2020).

[51] C. W. Helstrom, Quantum Detection and Estimation Theory (Springer, New York, 1969), Vol. 1, pp. 231-252.

[52] A. S. Holevo, Probabilistic and Statistical Aspects of Quantum Theory (Springer Science \& Business Media, New York, 2011), Vol. 1.

[53] S. L. Braunstein and C. M. Caves, Statistical Distance and the Geometry of Quantum States, Phys. Rev. Lett. 72, 3439 (1994).

[54] F. Binder, L. A. Correa, C. Gogolin, J. Anders, and G. Adesso, Thermodynamics in the Quantum Regime (Springer, New York, 2018), Vol. 195.

[55] S. Deffner and S. Campbell, Quantum Thermodynamics (Morgan \& Claypool Publishers, 2019), pp. 2053-2571.

[56] S. A. Frank and F. J. Bruggeman, The Fundamental Equations of Change in Statistical Ensembles and Biological Populations, Entropy 22, 1395 (2020).

[57] G. R. Price, Selection and Covariance, Nature (London) 227, 520 (1970).

[58] M. G. Paris, Quantum Estimation for Quantum Technology, Int. J. Quantum. Inform. 07, 125 (2009). 
[59] We show in Appendix B that when states with $p_{j}=0$ play a role in the evolution of an observable, there is a correction to Eq. (3) that is upper bounded by $\left|\sum_{p_{j}=0} \dot{p}_{j}\langle j|A| j\rangle\right|$. This correction is typically small for most times in a differentiable evolution and null for states with constant support.

[60] J. S. Sidhu and P. Kok, A Geometric Perspective on Quantum Parameter Estimation, AVS Quantum Sci. 2, 014701 (2020).

[61] M. Gessner and A. Smerzi, Statistical Speed of Quantum States: Generalized Quantum Fisher Information and Schatten Speed, Phys. Rev. A 97, 022109 (2018).

[62] We show in Appendix K4 that one can also derive a distinct speed limit [Eq. (K7)] from the classical CramérRao bound that results from restricting to measurements in the eigenbasis of observable $A$. However, this speed limit involves classical Fisher information that depends on the eigenbasis of the observable [Eq. (K5)].

[63] S. W. Flynn, H. C. Zhao, and J. R. Green, Measuring Disorder in Irreversible Decay Processes, J. Chem. Phys. 141, 104107 (2014).

[64] J. W. Nichols, S. W. Flynn, and J. R. Green, Order and Disorder in Irreversible Decay Processes, J. Chem. Phys. 142, 064113 (2015).

[65] A. Messiah, Quantum Mechanics (Dover Publications, New York, 1995).

[66] J. G. Muga, R. S. Mayato, and I. L. Egusquiza, Time in Quantum Mechanics (Springer, Berlin, 2007), Vol. 1.

[67] J. Anandan and Y. Aharonov, Geometry of Quantum Evolution, Phys. Rev. Lett. 65, 1697 (1990).

[68] J. Avron, R. Seiler, and L. Yaffe, Adiabatic Theorems and Applications to the Quantum Hall Effect, Commun. Math. Phys. 110, 33 (1987).

[69] D. Girolami, How Difficult Is It to Prepare a Quantum State?, Phys. Rev. Lett. 122, 010505 (2019).

[70] S. Alipour, A. Chenu, A. T. Rezakhani, and A. del Campo, Shortcuts to Adiabaticity in Driven Open Quantum Systems: Balanced Gain and Loss and Non-Markovian Evolution, Quantum 4, 336 (2020).

[71] L. Li, M. J. Hall, and H. M. Wiseman, Concepts of Quantum Non-Markovianity: A Hierarchy, Phys. Rep. 759, 1 (2018).

[72] K. Jacobs and D. A. Steck, A Straightforward Introduction to Continuous Quantum Measurement, Contemp. Phys. 47, 279 (2006).

[73] S. Cusumano and Ł. Rudnicki (private communication).

[74] Ł. Rudnicki and C. Gneiting, Stabilizable Gaussian States, Phys. Rev. A 98, 032120 (2018).

[75] U. Seifert, Stochastic Thermodynamics, Fluctuation Theorems and Molecular Machines, Rep. Prog. Phys. 75, 126001 (2012).

[76] M. Lostaglio, Certifying Quantum Signatures in Thermodynamics and Metrology via Contextuality of Quantum Linear Response, Phys. Rev. Lett. 125, 230603 (2020).

[77] Consider two qubits in a state with a spectral decomposition $\rho=p_{\text {ent }}\left|\Psi_{\text {ent }}\right\rangle\left\langle\Psi_{\text {ent }}\left|+p_{\text {prod }}\right| \Psi_{\text {prod }}\right\rangle\left\langle\Psi_{\text {prod }}\right|$, where $\left|\Psi_{\text {ent }}\right\rangle=(1 / \sqrt{2})(|00\rangle+|11\rangle)$ and $\left|\Psi_{\text {prod }}\right\rangle=|01\rangle$. A change in the probabilities $\left\{p_{\text {ent }}, p_{\text {prod }}\right\}$ has a description in terms of incoherent dynamics even though it involves a very quantum process where entanglement between the qubits can grow.

[78] R. A. Fisher, On the Mathematical Foundations of Theoretical Statistics, Philos. Trans. R. Soc. A 222, 309 (1922).

[79] E.-J. Kim, Investigating Information Geometry in Classical and Quantum Systems through Information Length, Entropy 20, 574 (2018).

[80] V. Vedral, The Role of Relative Entropy in Quantum Information Theory, Rev. Mod. Phys. 74, 197 (2002).

[81] M. Abramowitz, Handbook of Mathematical Functions, with Formulas, Graphs, and Mathematical Tables (Dover Publications, New York, 1974).

[82] S. Das, S. Khatri, G. Siopsis, and M. M. Wilde, Fundamental Limits on Quantum Dynamics Based on Entropy Change, J. Math. Phys. (N.Y.) 59, 012205 (2018).

[83] P. Boes, N. H. Y. Ng, and H. Wilming, The Variance of Relative Surprisal as Single-Shot Quantifier, arXiv: 2009.08391.

[84] D. Reeb and M. M. Wolf, Tight Bound on Relative Entropy by Entropy Difference, IEEE Trans. Inf. Theory 61, 1458 (2015).

[85] S. Bravyi, Upper Bounds on Entangling Rates of Bipartite Hamiltonians, Phys. Rev. A 76, 052319 (2007).

[86] M. Mariën, K. M. R. Audenaert, K. Van Acoleyen, and F. Verstraete, Entanglement Rates and the Stability of the Area Law for the Entanglement Entropy, Commun. Math. Phys. 346, 35 (2016).

[87] See Ref. [88] for a critical analysis of the interpretation of heat and work in open quantum systems.

[88] S. Alipour, A. T. Rezakhani, A. Chenu, A. del Campo, and T. Ala-Nissila, Unambiguous Formulation for Heat and Work in Arbitrary Quantum Evolution, arXiv:1912.01939.

[89] Note that $A_{C} \propto L_{C}$ and $A_{I} \propto L_{I}$ are sufficient but not necessary conditions to saturate the coherent and incoherent speed limits (10). For example, $A_{C} \propto L_{C}+\kappa_{C} \mathbb{1}$ and $A_{I} \propto L_{I}+\kappa_{I} \rrbracket$ also saturate them for constants $\kappa_{C}$ and $\kappa_{I}$, given that additive constants do not change covariances.

[90] W. K. Wootters, Statistical Distance and Hilbert Space, Phys. Rev. D 23, 357 (1981).

[91] I. Bengtsson and K. Zyczkowski, Geometry of Quantum States: An Introduction to Quantum Entanglement (Cambridge University Press, Cambridge, England, 2006).

[92] G. E. Crooks, Measuring Thermodynamic Length, Phys. Rev. Lett. 99, 100602 (2007).

[93] S. B. Nicholson, A. del Campo, and J. R. Green, Nonequilibrium Uncertainty Principle from Information Geometry, Phys. Rev. E 98, 032106 (2018).

[94] We show in Appendix J how to use the coherent-incoherent upper bound on observables to derive a tighter speed limit on the fidelity $F\left(\rho_{t}, \rho_{0}\right)$ between a pure initial state and the evolved state $\rho_{t}$.

[95] N. Gisin, G. Ribordy, W. Tittel, and H. Zbinden, Quantum Cryptography, Rev. Mod. Phys. 74, 145 (2002).

[96] S. Debnath, N. M. Linke, C. Figgatt, K. A. Landsman, K. Wright, and C. Monroe, Demonstration of a Small Programmable Quantum Computer with Atomic Qubits, Nature (London) 536, 63 (2016).

[97] J. Zhang, G. Pagano, P. W. Hess, A. Kyprianidis, P. Becker, H. Kaplan, A. V. Gorshkov, Z.-X. Gong, and C. Monroe, Observation of a Many-Body Dynamical Phase Transition 
with a 53-Qubit Quantum Simulator, Nature (London) 551, 601 (2017).

[98] F. Arute, K. Arya, R. Babbush, D. Bacon, J. C. Bardin, R. Barends, R. Biswas, S. Boixo, F. G. Brandao, D. A. Buell et al., Quantum Supremacy Using a Programmable Superconducting Processor, Nature (London) 574, 505 (2019).

[99] B. Hensen, H. Bernien, A. E. Dréau, A. Reiserer, N. Kalb, M. S. Blok, J. Ruitenberg, R. F. Vermeulen, R. N. Schouten, C. Abellán et al., Loophole-Free Bell Inequality Violation Using Electron Spins Separated by 1.3 Kilometres, Nature (London) 526, 682 (2015).

[100] M. Giustina et al., Significant-Loophole-Free Test of Bell's Theorem with Entangled Photons, Phys. Rev. Lett. 115, 250401 (2015).

[101] L. K. Shalm et al., Strong Loophole-Free Test of Local Realism, Phys. Rev. Lett. 115, 250402 (2015).

[102] J. Eisert, M. Friesdorf, and C. Gogolin, Quantum ManyBody Systems out of Equilibrium, Nat. Phys. 11, 124 (2015).

[103] C. Gogolin and J. Eisert, Equilibration, Thermalisation, and the Emergence of Statistical Mechanics in Closed Quantum Systems, Rep. Prog. Phys. 79, 056001 (2016).

[104] E. H. Lieb and D. W. Robinson, The Finite Group Velocity of Quantum Spin Systems, Commun. Math. Phys. 28, 251 (1972).
[105] M. Rigol, V. Dunjko, and M. Olshanii, Thermalization and Its Mechanism for Generic Isolated Quantum Systems, Nature (London) 452, 854 (2008).

[106] S. G. Schirmer, H. Fu, and A. I. Solomon, Complete Controllability of Quantum Systems, Phys. Rev. A 63, 063410 (2001).

[107] J. Werschnik and E. K. U. Gross, Quantum Optimal Control Theory, J. Phys. B 40, R175 (2007).

[108] M. Bukov, D. Sels, and A. Polkovnikov, Geometric Speed Limit of Accessible Many-Body State Preparation, Phys. Rev. X 9, 011034 (2019).

[109] P. M. Poggi, Analysis of Lower Bounds for Quantum Control Times and Their Relation to the Quantum Speed Limit, Anales AFA 31, 29 (2020).

[110] M. M. Wilde, Quantum Information Theory (Cambridge University Press, Cambridge, England, 2013).

[111] C. Helstrom, Minimum Mean-Squared Error of Estimates in Quantum Statistics, Phys. Lett. A 25, 101 (1967).

[112] G. Darmois, Sur les Limites de la Dispersion de Certaines Estimations, Revue de l'Institut International de Statistique 13, 9 (1945).

[113] H. Cramér, Mathematical Methods of Statistics (Princeton University, Princeton, NJ, 1946), p. 500.

[114] C. R. Rao, Information and the Accuracy Attainable in the Estimation of Statistical Parameters, in Breakthroughs in Statistics (Springer, New York, 1992), pp. 235-247. 